\title{
Guanine Nucleotide Exchange Factor for Eukaryotic Translation Initiation Factor 2 in Saccharomyces cerevisiae: Interactions between the Essential Subunits GCD2, GCD6, and GCD7 and the Regulatory Subunit GCN3
}

\author{
JANET L. BUSHMAN, MARCO FOIANI, $\dagger$ A. MARK CIGAN, $\ddagger$ CHRISTOPHER J. PADDON,§ AND \\ ALAN G. HINNEBUSCH* \\ Section on Molecular Genetics of Lower Eukaryotes, Laboratory of Molecular Genetics, National Institute of \\ Child Health and Human Development, Bethesda, Maryland 20892
}

Received 22 February 1993/Returned for modification 6 April 1993/Accepted 27 April 1993

\begin{abstract}
Phosphorylation of eukaryotic translation initiation factor 2 (eIF-2) in amino acid-starved cells of the yeast Saccharomyces cerevisiae reduces general protein synthesis but specifically stimulates translation of $G C N 4$ mRNA. This regulatory mechanism is dependent on the nonessential GCN3 protein and multiple essential proteins encoded by GCD genes. Previous genetic and biochemical experiments led to the conclusion that GCD1, GCD2, and GCN3 are components of the GCD complex, recently shown to be the yeast equivalent of the mammalian guanine nucleotide exchange factor for eIF-2, known as eIF-2B. In this report, we identify new constituents of the GCD-eIF-2B complex and probe interactions between its different subunits. Biochemical evidence is presented that GCN3 is an integral component of the GCD-eIF-2B complex that, while dispensable, can be mutationally altered to have a substantial inhibitory efiect on general translation initiation. The amino acid sequence changes for three $g c d 2$ mutations have been determined, and we describe several examples of mutual suppression involving the gcd2 mutations and particular alleles of $G C N 3$. These allele-specific interactions have led us to propose that GCN3 and GCD2 directly interact in the GCD-eIF-2B complex. Genetic evidence that GCD6 and GCD7 encode additional subunits of the GCD-eIF-2B complex was provided by the fact that reduced-function mutations in these genes are lethal in strains deleted for $G C N 3$, the same interaction described previously for mutations in $G C D 1$ and $G C D 2$. Biochemical experiments showing that GCD6 and GCD7 copurify and coimmunoprecipitate with GCD1, GCD2, GCN3, and subunits of eIF-2 have confirmed that GCD6 and GCD7 are subunits of the GCD-eIF-2B complex. The fact that all five subunits of yeast eIF-2B were first identified as translational regulators of $G C N 4$ strongly suggests that regulation of guanine nucleotide exchange on eIF-2 is a key control point for translation in yeast cells just as in mammalian cells.
\end{abstract}

Starvation of the budding yeast Saccharomyces cerevisiae for one or more amino acids leads to elevated transcription of more than 30 genes involved in amino acid biosynthesis. This response (general amino acid control) is mediated by an increase in the levels of the transcriptional activator protein GCN4 (reviewed in reference 25). The increase in GCN4 synthesis is coupled to amino acid starvation by a unique translational regulatory mechanism involving phosphorylation of the $\alpha$ subunit of eukaryotic translation initiation factor $2(\mathrm{eIF}-2 \alpha)$ by the protein kinase GCN2 (10; reviewed in reference 29). In mammalian cells, phosphorylation of eIF- $2 \alpha$ on the serine residue at position 51 inhibits translation in response to stress conditions, which include viral infection, amino acid starvation, and heat shock (reviewed in references 23 and 34 ). Recent results also implicate this regulatory mechanism in mammalian cell cycle control (32).

According to our current model, expression of GCN4 in $S$. cerevisiae is linked to the activity of eIF-2 by short upstream

\footnotetext{
${ }^{*}$ Corresponding author.

† Present address: Dipartimento di Genetica e di Biologia dei Microorganismi, Università degli Studi di Milano, 20133 Milan, Italy.

$¥$ Present address: Pioneer Hi-Bred, Johnston, IA 50131.

$\S$ Present address: Department of Molecular Science, Glaxo Group Research Limited, Greenford, Middlesex UB6 0HE, England.
}

open reading frames (uORFs) in the GCN4 mRNA leader (1, 10). It is thought that ribosomes scanning from the $5^{\prime}$ end of GCN4 mRNA translate the first uORF (uORF1) and resume scanning. Under conditions of sufficient eIF-2 activity, these ribosomes reinitiate translation at one of the remaining uORFs (uORF2 to uORF4) before reaching GCN4. Unlike uORF1, translation of these downstream uORFs does not allow subsequent reinitiation at the GCN4 AUG codon; therefore, GCN4 expression is repressed. When eIF-2 activity is reduced by mutations or by phosphorylation under conditions of amino acid starvation, many ribosomes scanning downstream from uORF1 cannot reinitiate at uORF2 to uORF4 and instead reinitiate further downstream at the GCN4 start codon. Thus, a decrease in the efficiency of reinitiation leads to an increase in GCN4 translation.

The principal role of eIF-2 in translation initiation is to deliver charged initiator tRNA $^{\text {Met }}$ to the ribosome (for reviews of eukaryotic translation initiation, see references 39 and 44). Analogous to elongation factor 1 in eukaryotes and elongation factor Tu in prokaryotes, the activity of eIF-2 is regulated by a cycle of GTP binding and hydrolysis. eIF-2 forms a ternary complex with GTP and tRNA $\mathrm{A}_{\mathrm{i}}^{\text {Met }}$ and subsequently binds to the $40 \mathrm{~S}$ ribosomal subunit. Upon recognition of the AUG start codon, the GTP is hydrolyzed and eIF-2 . GDP is released. In mammalian cells, a second initiation factor, known as eIF-2B, is required to promote GDP release and rebinding of GTP by eIF-2 (31). Phos- 
TABLE 1. Construction of plasmids for marker rescue mapping of $G C D 2$ alleles $^{a}$

\begin{tabular}{|c|c|c|c|c|}
\hline $\begin{array}{l}\text { pCP46-derived } \\
\text { plasmid }\end{array}$ & $\begin{array}{l}\text { YEp24-derived } \\
\text { plasmid }\end{array}$ & $\begin{array}{l}\text { Site used to create } \\
\text { deletions }\end{array}$ & $\begin{array}{l}\text { Oligonucleotide used to } \\
\text { generate in-frame deletions }\end{array}$ & $\begin{array}{l}\text { Nucleotides } \\
\text { deleted }^{b}\end{array}$ \\
\hline $\begin{array}{l}\text { pCP46 } \\
\text { pCP53 } \\
\text { pCP54 } \\
\text { pCP55 }\end{array}$ & $\begin{array}{l}\text { pCP57 } \\
\text { pCP58 } \\
\text { pCP59 } \\
\text { pCP60 }\end{array}$ & $\begin{array}{l}\text { None } \\
\text { PstI-Bam HI } \\
\text { PstI-NdeI } \\
\text { BamHI-EcoRI }\end{array}$ & $\begin{array}{l}\text { None } \\
\text { 5'-GATCCTGCA-3' } \\
\text { 5'-TATGCA-3' } \\
\text { 5'-GATCCATCCCAG-3' } \\
\text { 5'-AATTCTGGGATG-3' }\end{array}$ & $\begin{array}{c}\text { None } \\
158-940 \\
158-1283 \\
940-1970\end{array}$ \\
\hline pCP56 & pCP61 & NdeI-EcoRI & $\begin{array}{l}\text { 5'-TATGGTTCATCAG-3' } \\
5^{\prime} \text {-AATTCTGATGAACCA-3' }\end{array}$ & $1283-1970$ \\
\hline
\end{tabular}

\footnotetext{
${ }^{a}$ See text for details.
}

${ }^{b}$ Numbering relative to the 5' end of GCD2 mRNA (42).

phorylation of the $\alpha$ subunit of eIF- 2 on serine 51 inhibits the guanine nucleotide exchange activity of eIF-2B $(36,47,49)$, decreasing the level of eIF-2 . GTP and thereby inhibiting translation initiation.

In $S$. cerevisiae, replacement of serine 51 of eIF- $2 \alpha$ with alanine abolishes phosphorylation of eIF-2 by GCN2 and prevents the increase in GCN4 translation that normally accompanies amino acid starvation (10). By analogy with mammalian systems, phosphorylation of eIF-2 $\alpha$ in yeast cells is thought to diminish the rate of guanine nucleotide exchange on eIF-2 catalyzed by eIF-2B. The resulting decrease in levels of eIF-2 . GTP . Met-tRNA $A_{i}^{\text {Met }}$ ternary complexes would stimulate translation of GCN4 by the mechanism described above. Because GCN4 expression is sensitive to the level of eIF-2 activity $(10,53)$, mutations that affect the ability of eIF-2B to recycle eIF-2 . GDP to eIF2 . GTP should also impair translational control of $G C N 4$. Several lines of evidence indicate that previously identified trans-acting regulators of GCN4 are subunits of the yeast equivalent of mammalian eIF-2B. Three such factors, GCD1, GCD2, and GCN3, were found to be components of a high-molecular-weight complex (the GCD complex) that resembles mammalian eIF-2B in being associated with a fraction of the eIF-2 in the cell (6). Recent biochemical experiments indicate that this GCD complex has the guanine nucleotide exchange activity characteristic of mammalian eIF-2B (5). In accord with the idea that a reduction in eIF-2B activity is responsible for increased translation of $G C N 4$, reduced-function mutations in $G C D 1$ and $G C D 2$ lead to high-level GCN4 translation, independent of GCN2 kinase function (25).

Our recent analysis of the GCD6 and GCD7 genes led us to propose that they encode additional subunits of the yeast GCD-eIF-2B complex (4). Reduced-function mutations in either gene lead to constitutive derepression of GCN4 translation independently of $G C N 2$, and deletion of each gene is lethal. Analysis of the cloned genes revealed significant amino acid sequence similarities between GCD7 and the GCN3 and GCD2 subunits of the GCD-eIF-2B complex and between GCD6 and GCD1. In addition, we discovered strong sequence similarity between GCD6 and the largest subunit of rabbit eIF-2B (4). We chose to interpret these mutant phenotypes and sequence similarities as evidence that GCD6 and GCD7 are integral subunits of the GCDeIF-2B complex. An alternative possibility is that the sequence relatedness between the proteins reflects redundancy of function and that multiple GCD complexes which contain exclusively GCD2, GCD7, or GCN3 and either GCD1 or GCD6 could exist.

In this report, we provide genetic and biochemical evidence that GCD6, GCD7, and GCN3 occur largely, if not entirely, as subunits of the same complexes which contain GCD1, GCD2, and a fraction of eIF-2. We demonstrate that the dispensable subunit GCN3 can be mutated to have strong effects on general protein synthesis in addition to affecting the translational control of GCN4. Finally, we describe several cases of mutual suppression involving mutations in $G C N 3, G C D 2$, and $G C D 7$. These genetic interactions, combined with the sequence similarities among GCN3, GCD2, and GCD7, lead us to suggest that these three related proteins interact with one another in the GCD-eIF-2B complex and play an important role in its regulation by phosphorylated eIF-2.

\section{MATERIALS AND METHODS}

Polysome profile analysis. Preparation and gradient analysis of yeast polysomes were performed essentially as described previously (12) except that cultures were grown at $30^{\circ} \mathrm{C}$ in YEPD or SD medium (48) as indicated. Single-copy plasmids bearing $G C N 3$ alleles were described previously (17). Immunoblot analysis of gradient fractions for the $\beta$ subunit of eIF-2 was performed as described for eIF-2 $\alpha$ (12) except that eIF-2 $\beta$ antibodies (6) were used. The Pet ${ }^{-}$ derivative of strain $\mathrm{H} 1402$ used as a control in these experiments was generated by growing $\mathrm{H} 1402$ in the presence of $10 \mu \mathrm{g}$ of ethidium bromide per $\mathrm{ml}$ and screening for respiratory-deficient colonies on YEPG plates (48).

Construction of plasmids used for marker rescue mapping of $g c d 2$ mutations. High-copy-number plasmids containing $G C D 2$ or derivatives thereof were constructed as follows. A SalI-EagI fragment containing GCD2 was isolated from pCP46 (43) and inserted between the SalI and EagI sites of YEp24 (45), generating pCP57. gcd2 genes lacking internal portions of the ORF were generated by restriction enzyme digestion of pCP46 followed by religation in the presence of synthetic oligonucleotides designed to produce in-frame deletions. The deletion junctions were sequenced, and the deletion alleles were subcloned into YEp24 on SalI-EagI fragments as described for pCP57. The construction of these plasmids is summarized in Table 1.

Cloning and nucleotide sequence analysis of $\mathrm{gcd} 2$ alleles. (i) gcd2-502. A 450-bp fragment containing the 340-bp BamHINdeI region in GCD2 was amplified from genomic DNA of strain $\mathrm{H} 63$ isolated as described by Winston et al. (54) by using the polymerase chain reaction (PCR) and oligonucleotide primers $6\left(5^{\prime}\right.$-ATTATCTAGAGCAGTAACAGTTCAG TTAGAACC-3') and 7 (5'-AAAAGAATTCGAATAAAGA CCTTATCCATCCGTCG-3'). The BamHI-NdeI fragment from the amplified sequence was inserted between the BamHI and NdeI sites of pCP46 to form plasmid pMF11. DNA sequencing of both strands of two independent isolates 
of the amplified BamHI-NdeI region revealed a single-point mutation (AGA to GGA) that changes arginine 334 to glycine in GCD2.

(ii) gcd2-503. The 450-bp fragment described above containing the 340-bp Bam HI-NdeI region in GCD2 was PCR amplified from genomic DNA of strain H64, using primers 6 and 7 described above. The amplified fragment was digested with $X b a I$ and EcoRI and cloned between the $X b a I$ and EcoRI sites of pBLUESCRIPTsk to form plasmid pMF20. DNA sequencing of both strands of two independent isolates of the amplified BamHI-NdeI region revealed a single-point mutation (AGA to AGT) that changes arginine 334 to serine in GCD2.

(iii) gcd2-1. An 850-bp fragment containing the 685-bp NdeI-EcoRI region was PCR amplified from genomic DNA of strain H954, using primers 4 (5'-TTAAGAATTCATT GACCCATCTACCCCCGACAAAG-3') and 5 (5'-ACAGG GACAGATGAAGGTGGCAAAG-3'). The amplified fragment was digested with EcoRI and cloned into the EcoRI site of pBLUESCRIPTks for sequencing. The only clones obtained containing the amplified fragment had the $3^{\prime}$ end of the fragment fused to the vector through the primer 5 used for amplification. DNA sequencing of both strands $3^{\prime}$ to the NdeI site of two independent isolates of the amplified fragment revealed a single-point mutation (GAA to TAA) that changes glutamate 631 to a stop codon, creating a 21-amino-acid truncation of GCD2. This change destroys the EcoRI site in the $3^{\prime}$ end of GCD2 that was being used to subclone the amplified fragments, thus accounting for the products that we recovered.

Genetic methods and yeast strain constructions. Standard techniques for growth, genetic analysis, and plasmid transformation of yeast strains were performed as described elsewhere $(30,48)$. Resistance to 3-aminotriazole (3-AT), an inhibitor of the HIS 3 product (25), was determined by replica plating to SD medium containing $30 \mathrm{mM}$ 3-AT as described previously (27). Strains having inducible or constitutively elevated GCN4 expression are resistant to 3-AT (3-AT $\left.{ }^{r}\right)$ because GCN4 activates transcription of genes encoding histidine biosynthetic enzymes. gcn 2 or gcn 3 mutant strains are uninducible and thus sensitive to 3-AT (3-AT $\left.{ }^{\mathrm{s}}\right)$. Resistance to 5-fluoro-DL-tryptophan (5-FT) was determined by replica plating on SD medium containing $0.5 \mathrm{mM} \mathrm{5-FT}$ as described previously (53). Because 5-FT is toxic unless tryptophan levels are elevated but does not cause an amino acid starvation signal, only strains with constitutively elevated expression of GCN4 are resistant to this analog (5-FT $)$; thus, wild-type and gcn strains are sensitive to 5-FT $\left(5-\mathrm{FT}^{\mathrm{s}}\right)$.

Yeast strains used in this study (Table 2) are congenic to the wild-type strain S288C. Strain EY462 was constructed by replacement of the gcn3-101 allele in $\mathrm{H} 1456$ with the gcn3::LEU2 allele isolated from Ep308 as described previously (17). gcd6-1 strain H1597 was generated from successive backcrosses of F222 (MATa gcd6-1 leu2-2) (40) to $\mathrm{H750}$ (MATa gcn2::LEU2 leu2-3 leu2-112 ura3-52) or H751 (MAT $\alpha$ gcn2::LEU2 leu2-3 leu2-112 ura3-52) as described previously for gcd6-1 strains H1916 and H1917 (4). gcn3-102 GCD6:: $U R A 3$ strain $\mathrm{H} 1883$ was an ascospore clone from a cross between gcn3-102 strain $\mathrm{H} 51$ and GCD6::URA3 strain $\mathrm{H} 1724$. The URA3-marked allele of GCD6 in $\mathrm{H} 1724$ was generated by integration of $U R A 3$ GCD6 plasmid pJB98 at gcd6-1; $\mathrm{H1724}$ was the $\mathrm{Ura}^{+} \mathrm{Gcd}^{+}$transformant from which the 5-fluoro-orotic acid-resistant (3) $\left(\mathrm{Ura}^{-}\right)$strains $\mathrm{H} 1728$ and $\mathrm{H} 1730$ were derived as described previously (4). gcn $3^{c}$. R104K strains $\mathrm{H} 1499$ and $\mathrm{H} 1504$ were generated as de- scribed for H1489 (17). H1724 was crossed to $g c n 3^{\mathrm{c}}$ - $R 104 \mathrm{~K}$ strain $\mathrm{H} 1504$ to produce $\mathrm{H} 2114$ and $\mathrm{H} 2115$. The URA3marked allele of $G C D 7$ was generated previously by integration of plasmid pJB87(GCD7 URA3) at $G C D 7$ in strain $\mathrm{H} 750$ as described; the resulting strain H1834 was used for the genetic demonstration that GCD7 had been cloned (4). GCD7::URA3 strain $\mathrm{H} 1834$ was crossed to gcn3-102 strain $\mathrm{H} 17$ to generate $\mathrm{H} 1839$. $\mathrm{H} 1834$ was crossed to $g c n 3^{\mathrm{c}}-\mathrm{R} 104 \mathrm{~K}$ strain $\mathrm{H} 1499$ to generate $\mathrm{H} 1840$.

Double-mutant combinations to test the interactions between gcd6-1 or gcd7-201 and different alleles of $G C N 3$ were generated and analyzed as follows.

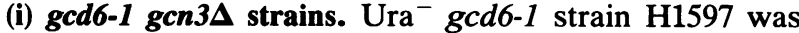
crossed to gcn3::URA3 strain EY162, and 26 tetrads were analyzed. Phenotypes of slow growth, 3-AT ${ }^{\mathrm{r}}$, and $5-\mathrm{FT}^{\mathrm{r}}$ were used to monitor segregation of the gcd6-1 allele; $\mathrm{Ura}^{+}$ and 3-ATs phenotypes were used to identify the gcn3::URA3 allele. Many ascospore clones failed to grow or produced pinpoint colonies after 2 weeks. Nine tetrads had two viable colonies, 14 tetrads had three viable colonies, and 3 tetrads had four viable colonies. No viable gcd6-1 gcn3::URA3 double mutants were recovered, and the nonviable ascospores were predicted to contain both gcn3::URA3 and gcd6-1, as judged from the segregation of the phenotypes described above in the viable colonies. From these results, we concluded that gcd6-1 gcn3::URA3 double mutants are inviable.

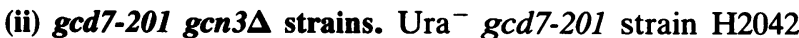
was crossed to gcn3::URA3 strain EY162, and 29 tetrads were analyzed. Similar to the results obtained with gcd6-1, many ascospore clones failed to grow or produced pinpoint colonies after 2 weeks. Nine tetrads had two viable colonies, 15 tetrads had three viable colonies, and 4 tetrads had four viable colonies. The nonviable ascospores were predicted to contain both gcn3::URA3 and gcd7-201, as judged from the phenotypes of the viable colonies. From these results, we concluded that gcd7-201 gcn3::URA3 double mutants are inviable.

(iii) gcd6-1 gcn3-102 strains. gcd6-1 GCN3 strain H1728 was crossed to GCD6::URA3 gcn3-102 strain $\mathrm{H} 1883$, and 12 complete tetrads were analyzed. The presence of gcd6-1 was inferred on the basis of uracil auxotrophy, and the presence of gcn3-102 was determined by complementation tests with gcn $3 \Delta$ strains $\mathrm{H} 741$ and $\mathrm{H} 742$. We observed no differences between the gcd6-1 GCN3 and the gcd6-1 gcn3-102 colonies for growth rate, 3-AT ${ }^{\mathrm{T}}$, or $5-\mathrm{FT}^{\mathrm{T}}$ phenotype. From these results, we concluded that gcd6-1 is epistatic to gcn3-102.

(iv) gcd7-201 gcn3-102 strains. gcd7-201 GCN3 strain $\mathrm{H} 1603$ was crossed to GCD7::URA3 gcn3-102 strain $\mathrm{H} 1839$, and 21 complete tetrads were analyzed. The gcd7-201 colonies were identified on the basis of uracil auxotrophy, and the location of gcn3-102 was determined as described above. We observed three distinct spore colony sizes in these tetrads. The class containing large colonies was composed of wild-type GCD7::URA3 GCN3 and double-mutant gcd7-201 gcn3-102 ascospore clones. It was remarkable that the double-mutant colonies grew as well as did the wild-type colonies, since gcd7-201 typically causes a significant slowgrowth phenotype. The wild-type colonies were more resistant to 3-AT than were the double-mutant colonies. The class of medium-size colonies was composed of GCD7::URA3 gcn3-102 ascospore clones, which grew as well as the previous class when streaked for single colonies on YEPD. Possibly, these gcn3 spores were slow to germinate because of amino acid imbalances or deficiencies that would otherwise have been corrected by the general amino 
TABLE 2. Yeast strains used in this study

\begin{tabular}{|c|c|c|}
\hline Strain & Genotype & Source or reference \\
\hline EY162 & MAT $\alpha$ gcn3::URA3 trp1 ura3-52 leu2-3 leu2-112 & E. Hannig \\
\hline EY448 (H1426) & MAT $\alpha$ gcn3::LEU2 inol ura3-52 leu2-3 leu2-112 HIS4-lacZ & 17 \\
\hline EY462 (F395) & MATa gcd2-502 gcn3::LEU2 ura3-52 leu2-3 leu2-112 & This study \\
\hline MC1057 & MATa gcd1::LEU2 trp1D63 ura3-52 leu2-3 leu2-112 HIS4-lacZ <pBM31(GCD1-HA TRP1)> & \\
\hline CP24 & MAT $\alpha$ gcd2-1 gcn2::LEU2 & C. Paddon \\
\hline F222 & MATa gcd6-1 leu2-2 & 40 \\
\hline H17 & MAT $\alpha$ gcn3-102 ura3-52 leu2-3 leu2-112 & 19 \\
\hline H51 & MATa gcn3-102 ura3-52 HIS4-lacZ & Hinnebusch laboratory \\
\hline H63 & MAT $\alpha$ his1-29 gcn2-101 gcn3-101 gcd2-502 ura3-52 HIS4-lacZ & 21 \\
\hline H64 & MATa his1-29 gcn2-101 gcn3-101 gcd2-503 ura3-52 HIS4-lacZ & 21 \\
\hline H464 & MAT $\alpha$ gcn3-101 gcn2-101 gcd2-502 & S. Harashima \\
\hline H630 & MATa gcn3-101 gcd2-502 ura3-52 leu2-3 leu2-112 & 20 \\
\hline H645 & MATa gcn3-101 gcn2-101 gcd2-503 & S. Harashima \\
\hline H652 & $M A T \alpha$ gcd2-503 ura3-52 leu2-3 leu2-112 & 20 \\
\hline H741 & MAT $\alpha$ gcn3::LEU2 lys2 leu2-3 leu2-112 & 43 \\
\hline H742 & MATa gcn3::LEU2 lys2 leu2-3 leu2-112 & 43 \\
\hline H750 & MATa gcn2::LEU2 leu2-3 leu2-112 ura3-52 & 33 \\
\hline H751 & MAT $\alpha$ gcn2::LEU2 leu2-3 leu2-112 ura3-52 & 33 \\
\hline H954 & MATa gcd2-1 gcn3::LEU2 ura3-52 leu2-3 leu2-112 & 43 \\
\hline H1333 & MATa gcn2::URA3 inol ura3-52 leu2-3 leu2-112 HIS4-lacZ & 17 \\
\hline H1402 & $M A T \alpha$ ino1 ura3-52 leu2-3 leu2-112 HIS4-lacZ & 17 \\
\hline H1402Pet ${ }^{-}$ & MATa ino1 ura3-52 leu2-3 leu2-112 HIS4-lacZ & This study \\
\hline H1456 & MATa gcd2-502 gcn3-101 ura3-52 leu2-3 leu2-112 & 12 \\
\hline H1489 & MAT $\alpha$ gcn3 $3^{c}-R 104 K$ inol ura3-52 leu2-3 leu2-112 HIS4-lacZ & 17 \\
\hline H1491 & MAT $\alpha$ gcn $3^{c}-A 26 T$ ino1 ura3-52 leu2-3 leu2-112 HIS4-lacZ & 17 \\
\hline H1499 & $M A T \alpha$ gcn $3^{c}-R 104 K$ ura3-52 leu2-3 leu2-112 & This study \\
\hline H1504 & MATa gcn3 ${ }^{\mathrm{c}}-\mathrm{R} 104 \mathrm{~K}$ ino1 ura3-52 leu2-3 leu2-112 HIS4-lacZ & This study \\
\hline H1597 & MATa gcd6-1 gcn2::LEU2 ura3-52 leu2-3 leu2-112 & This study \\
\hline H1603 & MATa gcd7-201 ura3-52 leu2-3 leu2-112 & 4 \\
\hline H1724 & $M A T \alpha$ GCD6::URA3 ura3-52 leu2-3 leu2-112 & This study \\
\hline H1727 & MAT $\alpha$ ura3-52 leu2-3 leu2-112 & 4 \\
\hline H1728 & MAT $\mathrm{gcd6}-1$ ura3-52 leu2-3 leu2-112 & 4 \\
\hline H1730 & $M A T \alpha$ ura3-52 leu2-3 leu2-112 & 4 \\
\hline H1834 & MATa gcn2::LEU2 GCD7::URA3 ura3-52 leu2-3 leu2-112 & This study \\
\hline H1839 & MATa gcn3-102 GCD7::URA3 ura3-52 leu2-3 leu2-112 & This study \\
\hline H1840 & MATa gcn3 ${ }^{\mathrm{c}}-\mathrm{R} 104 \mathrm{~K}$ GCD7::URA3 ura3-52 leu2-3 leu2-112 & This study \\
\hline H1872 & MAT $\alpha$ gcn3-102 ura3-52 leu2-3 leu2-112 & This study \\
\hline H1873 & MATa gcn3-102 gcd7-201 ura3-52 leu2-3 leu2-112 & This study \\
\hline H1875 & MATa ura3-52 leu2-3 leu2-112 & This study \\
\hline H1876 & MATa gcd7-201 ura3-52 leu2-3 leu2-112 & This study \\
\hline H1877 & MATa gcn3-102 gcd7-201 ura3-52 leu2-3 leu2-112 & This study \\
\hline H1879 & MAT $\alpha$ gcn3-102 ura3-52 leu2-3 leu2-112 & This study \\
\hline H1880 & MATa gcd7-201 ura3-52 leu2-3 leu2-112 & This study \\
\hline H1882 & MATa ura3-52 leu2-3 leu2-112 & This study \\
\hline H1883 & MATa gcn3-102 GCD6::URA3 ura3-52 HIS4-lacZ & This study \\
\hline $\mathrm{H} 2042$ & MATa gcn2::LEU2 gcd7-201 ura3-52 leu2-3 leu2-112 & 4 \\
\hline H2114 & MATa gcn3'-R104K GCD6::URA3 ura3-52 leu2-3 leu2-112 HIS4-lacZ & This study \\
\hline $\mathrm{H} 2115$ & MATa gcn $3^{3}-R 104 K$ GCD6::URA3 ura3-52 HIS4-lacZ & This study \\
\hline
\end{tabular}

acid control pathway; growth defects on rich media have not previously been observed in gcn3 strains. As expected, these gcn3-102 colonies were $3-\mathrm{AT}^{\mathrm{s}}$. Finally, the class containing the smallest colonies was composed of gcd7-201 GCN3 ascospore clones. These strains retained the slowgrowth phenotype when streaked for single colonies on YEPD and were resistant to 3-AT and 5-FT, as expected for gcd7-201 mutants. These results indicate that gcn3-102 fully suppresses the slow-growth phenotype of gcd7-201 and that gcd7-201 partially suppresses the 3-AT sensitivity associated with gcn3-102.

(v) gcd6-1 gcn 3c-R104K strains. gcd6-1 strain H1728 was crossed to GCD6::URA3 gcn $3^{\circ}-R 104 K$ strains $\mathrm{H} 2114$ and $\mathrm{H} 2115$, and a total of 28 tetrads were analyzed. Many ascospores were inviable; pinpoint colonies were not produced in this cross. The inviable ascospores were predicted to contain both $g c d 6-1$ and $g c n 3^{\mathrm{c}}-\mathrm{R} 104 \mathrm{~K}$, indicating that gcd6-1 gcn $3^{c}-R 104 K$ double mutants are inviable.

(vi) gcd7-201 gcn3 - R104K strains. gcd7-201 strain H1603 was crossed to GCD7::URA3 gcn3 ${ }^{\mathrm{C}}$-R104K strain $\mathrm{H} 1840$, and 22 tetrads were analyzed. As with gcd6-1, many of the ascospores were inviable, and these were predicted to contain both $g c d 7-201$ and $g c n 3^{c}-R 104 K$. This finding indicates that $g c d 7-201$ gcn $3^{\mathrm{c}}-\mathrm{R} 104 \mathrm{~K}$ double mutants are also inviable.

Assays of GCN4-lacZ expression. The GCN4-lacZ fusion present on the low-copy-number $U R A 3$ plasmid p180 (24) was introduced into all four ascospore clones from two tetratype tetrads obtained from the cross between $\mathrm{H} 1603$ (gcd7-201 GCN3) and H1839 (GCD7::URA3 gcn3-102). To achieve this, 5-fluoro-orotic acid selection (3) was used beforehand to obtain $\mathrm{Ura}^{-}$derivatives of the GCD7::URA3 
ascospore clones. Such colonies were readily obtained because $U R A 3$ is flanked by tandem repeats of the wild-type $G C D 7$ gene in the GCD7::URA3 allele. The GCN4-lacZ fusion on p180 has the wild-type leader containing all four uORFs and the amino-terminal coding region of $G C N 4$ fused to $\operatorname{lacZ}$. $\beta$-Galactosidase assays were conducted as described elsewhere (38).

GCD6 and GCD7 antibody production. TrpE-GCD6 and TrpE-GCD7 fusion proteins isolated from Escherichia coli were used to raise antibodies directed against GCD6 and GCD7, respectively. The TrpE-GCD6 expression plasmid pJB77 was constructed by inserting the 4-kb BglII-HindIII fragment from pJB6 between the Bam HI and HindIII sites of pATH3 (11) (pJB6 contains the 8.6-kb HindIII fragment from pJB1 [4] inserted at the HindIII site of pRS316 [50]). This produces an in-frame fusion of TrpE with amino acids +121 to +712 of GCD6. The TrpE-GCD7 expression plasmid pJB106 was constructed by inserting the 1.1-kb $X b a I$ fragment of pJB99 (4) into the XbaI site of pATH2 (11). This produces an in-frame fusion of $\operatorname{TrpE}$ with amino acids +74 to +381 of GCD7. Rabbits were injected with 0.5 to $1.0 \mathrm{mg}$ of the respective fusion proteins and boosted at 4-week and, subsequently, 2-week intervals by Hazleton Laboratories.

High-copy-number $G C D 6$ and $G C D 7$ plasmids. pJB115, a high-copy-number $L E U 2$ plasmid containing GCD6, was constructed by inserting the 3-kb SpeI fragment from pJB5 into the SpeI site located in the multiple cloning site of pRS425 (50). In this construct, the BamHI site in the multiple cloning site of the vector is 5' of GCD6. pJB111, a high-copy-number $U R A 3$ plasmid containing $G C D 7$, was constructed by inserting the 2.1-kb EagI-SalI fragment of pJB99 between the EagI and SalI sites of YEp24 (45).

Immunoprecipitation of the GCD-eIF-2 complex. Subunits of the GCD complex were immunoprecipitated from ribosomal salt wash (RSW) extracts prepared from exponentially growing yeast cells and analyzed essentially as described previously (6). The GCD6- and GCD7-specific antibodies were used at dilutions of 1:500 and 1:250, respectively (6).

\section{RESULTS}

gcn $3^{c}$ alleles impair general translation initiation. Unlike mutations affecting the GCD1 or GCD2 subunit of the GCD-eIF-2B complex, deletion of GCN3 does not result in an obvious general translation defect, even though GCN3 is associated with the complex $(5,6,42)$. We have described numerous mutations in $G C N 3$, called $g c n 3^{c}$ alleles, that result in constitutive derepression of $G C N 4$ translation. In addition to this regulatory phenotype, many $g c n 3^{c}$ alleles reduce the growth rate of cells (17). It seemed likely that the slow-growth phenotype of the gcn $3^{c}$ mutations results from an inhibitory effect of the mutant GCN3 proteins on the essential function of the GCD-eIF-2B complex in translational initiation. To address this possibility, we introduced $g c n 3^{c}$ alleles that confer slow growth rates (17) into a strain deleted for the chromosomal copy of GCN3 and analyzed the total polysome profiles of the resulting transformants by velocity sedimentation of whole cell extracts on sucrose gradients.

The results shown in Fig. 1A and Table 3 indicate that the $g c n 3^{\mathrm{c}}$ alleles with the greatest growth defects $\left(g c n 3^{\mathrm{c}}-A 26 T\right.$, $g c n 3^{c}-R 104 K, g c n 3^{c}-E 199 K$, and $\left.g c n 3^{c}-V 295 F\right)$ lead to an increase in the ratio of monosomes to polysomes by a factor ranging from 1.3 to 2.0. An increase in the monosome/ polysome ratio was reported previously for the $g c d 1-101$ (6) and $g c d 2-503$ (12) mutations and is indicative of a reduction

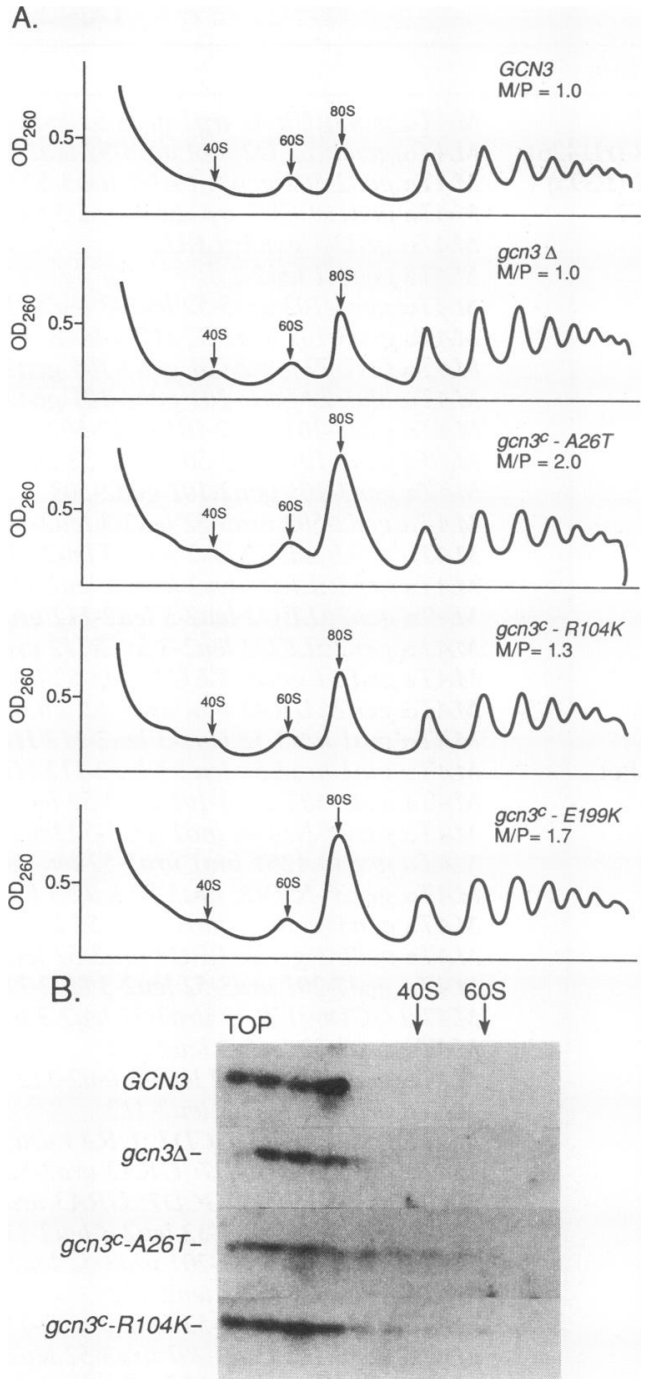

FIG. 1. Effects of $g c n 3^{c}$ alleles on general translation revealed by analysis of polysome profiles. (A) Single-copy plasmids bearing the GCN3 alleles indicated in each panel were introduced into gcn3s strain $\mathrm{H} 1426$. Extracts prepared from the resulting transformants grown to exponential phase at $30^{\circ} \mathrm{C}$ in $\mathrm{SD}$ medium were separated by centrifugation on low-salt 7 to $47 \%$ sucrose gradients for $2.5 \mathrm{~h}$ at $270,000 \times g$. Gradients were scanned at $260 \mathrm{~nm}$ while fractions were collected, and the absorbance profiles are shown, with the top of each gradient on the left. Positions of $40 \mathrm{~S}, 60 \mathrm{~S}$, and $80 \mathrm{~S}$ ribosomal species are indicated. For each profile, the areas under the $80 \mathrm{~S}$ (monosome) peak and the polysome peaks were calculated, and the ratio of monosome to polysome areas $(\mathrm{M} / \mathrm{P})$ was normalized so that the ratio for the $G C N 3$ transformant is 1.0 . $\mathrm{OD}_{260}$, optical density at $260 \mathrm{~nm}$. (B) Distribution of eIF-2 in polysome profiles. Extracts were prepared from GCN3 (H1402), gcn3A (H1426), gcn3 ${ }^{c}-A 26 T$ (H1491), and $g c n 3^{\mathrm{c}}-\mathrm{R} 104 \mathrm{~K}(\mathrm{H} 1489)$ isogenic yeast strains grown to exponential phase at $30^{\circ} \mathrm{C}$ in YEPD medium, and polysomes were separated on sucrose gradients as described above. Gradient fractions were collected, and proteins were precipitated, subjected to SDS-PAGE (10\% gel), and analyzed by immunoblotting with antibodies against eIF-2 $\beta$ according to the methods described for detection of eIF- $2 \alpha$ in polysome gradient fractions (12). The first lane on the left corresponds to the top fraction of each gradient, and the positions of $40 \mathrm{~S}$ and $60 \mathrm{~S}$ peaks in the fractions are indicated above. Relevant genotypes are shown on the left. The monosome/ polysome ratios (normalized to the ratio for the GCN3 strain) observed for these gradients were as follows: $g c n 3 \Delta, 1.0 ; \operatorname{gcn} 3^{c}$ $A 26 T, 1.8$; and $g c n 3^{\mathrm{c}}-\mathrm{R} 104 \mathrm{~K}, 1.4$. 
TABLE 3. Monosome/polysome ratios in $g c n 3^{c}$ mutant extracts

\begin{tabular}{|c|c|c|}
\hline Plasmid-borne allele ${ }^{a}$ & Growth rate ${ }^{b}$ & $\mathrm{M} / \mathrm{P}^{c}$ \\
\hline Vector $(g c n 3 \Delta)$ & ++++ & 1.0 \\
\hline$G C N 3$ & ++++ & 1.0 \\
\hline $\operatorname{gcn} 3^{\mathrm{c}}-A 26 T$ & + & 2.0 \\
\hline$g c n 3^{c}-S 65 F$ & $++^{+}$ & 0.96 \\
\hline $\operatorname{gcn} 3^{\mathrm{c}}-D 71 N$ & +++ & 0.93 \\
\hline$g c n 3^{\mathrm{c}}-R 104 \mathrm{~K}$ & ++ & 1.3 \\
\hline gcn $3^{\mathrm{c}}-E 199 K$ & + & 1.7 \\
\hline$g c n 3^{c}-V 295 F$ & + & 1.6 \\
\hline
\end{tabular}

${ }^{a}$ Single-copy plasmids derived from vector YCp50 bearing the designated $G C N 3$ alleles (17) were introduced into gcn3D strain H1426.

$b$ Assessed by examining colony size 3 to 7 days after cells were streaked on SD medium and incubated at $30^{\circ} \mathrm{C}$. A superscript plus indicates less growth than that signified by a normal plus.

${ }^{c}$ The ratio of monosomes to polysomes $(\mathrm{M} / \mathrm{P})$ was calculated from data of the type shown in Fig. 1 and normalized so that the ratio for the strain bearing wild-type $G C N 3$ is 1.0. Growth of strains and polysome analysis are described in the legend to Fig. 1 and in Materials and Methods.

in the rate of translation initiation relative to translation elongation. The parental strain lacking GCN3 was indistinguishable from the wild-type GCN3 transformant in growth rate and polysome content. To address the possibility that the decrease in polysome content seen in the $g c n 3^{c}$ mutants is an indirect effect of slow growth, we determined the polysome profile of an isogenic petite mutant containing wild-type GCN3 that grows slowly because of respiratory deficiency. This strain had a normal monosome/polysome ratio of 1.0 despite its slow growth rate (data not shown).

The $g c d 1$ and $g c d 2$ mutants mentioned above that exhibited a reduction of polysome content were found previously to contain increased amounts of eIF-2 migrating with small ribosomal subunits, suggesting an accumulation of $43 \mathrm{~S}$ or $48 \mathrm{~S}$ translational initiation intermediates $(6,12)$. A similar result was obtained here (Fig. 1B) for the $g c n 3^{c}-A 26 T$ and gcn $3^{\mathrm{c}}-\mathrm{R} 104 \mathrm{~K}$ mutants. Cell extracts were fractionated on sucrose gradients, and antibodies specific for the $\beta$ subunit of eIF-2 were used for immunoblot analysis to determine the eIF-2 content of each gradient fraction. Whereas the parental gcn $3 \Delta$ strain and the GCN3 transformant had no detectable eIF-2 $\beta$ in the $40 S-60 S$ region of the gradient, the $g c n 3^{c}$ mutants exhibited significant amounts of eIF- $2 \beta$ distributed in these fractions, particularly for the slowest-growing $g c n 3^{c}-A 26 T$ strain. Taken together, the results shown in Fig. 1 suggest that $g c n 3^{c}$ mutants have defects in general translation initiation similar to those present in gcd1-101 and gcd2-503 strains, consistent with the idea that GCN3 is an integral component of the essential GCD-eIF-2B complex of S. cerevisiae $(5,6)$.

Sequence of three $G C D 2$ mutations showing different genetic interactions with GCN3. Harashima et al. (20) reported that the gcd2-502 and $g c d 2-503$ mutations are conditionally lethal in the presence of gcn3-101 or a gcn3 deletion but not in strains containing wild-type GCN3. In contrast, the slowgrowth and temperature-sensitive phenotypes of the gcd2-1 mutation were found to be relatively unaffected by the presence or absence of GCN3 (43) (see Table 5). Given the fact that GCD2 and GCN3 exhibit regions of significant sequence similarity, one hypothesis to explain the ability of GCN3 to suppress certain gcd 2 mutations but not others was that the gcd2-502 and gcd2-503 mutations alter amino acids in regions of sequence similarity between GCD2 and GCN3 such that GCN3 can functionally substitute for these mutant gcd 2 proteins. In contrast, the gcd2-1 mutation would affect a region unrelated to GCN3 in both sequence and function,
TABLE 4. Marker rescue mapping of $g c d 2$ mutations $^{a}$

\begin{tabular}{|c|c|c|c|c|}
\hline \multirow{2}{*}{ Plasmid } & \multicolumn{4}{|c|}{$\begin{array}{l}\text { Formation of temperature-resistant papillae } \\
\text { by transformants of strain: }\end{array}$} \\
\hline & $\begin{array}{c}\mathrm{H630} \\
(\mathrm{gcd} 2-502)\end{array}$ & $\begin{array}{c}\mathrm{H} 464 \\
(\operatorname{gcd} 2-502)\end{array}$ & $\begin{array}{c}\mathrm{H} 645 \\
(\operatorname{gcd} 2-503)\end{array}$ & $\begin{array}{c}\text { CP24 } \\
(\operatorname{gcd} 2-1)\end{array}$ \\
\hline pCP58 (gcd2- $\Delta 158-940)$ & + & + & + & + \\
\hline pCP59 (gcd2- $\Delta 158-1283)$ & - & - & $(-)^{b}$ & + \\
\hline pCP60 $(g c d 2-\Delta 940-1970)$ & - & - & - & - \\
\hline pCP61 $(g c d 2-\Delta 1283-1970)$ & + & + & + & - \\
\hline YEp24 (vector) & - & - & - & - \\
\hline
\end{tabular}

a Strains H630, H464, H645, and CP24 were transformed with the indicated plasmids, and four independent transformants for each plasmid were tested for the ability to produce papillae at $36^{\circ} \mathrm{C}$. A plus sign indicates the formation of 5 to 20 papillae in each of four independent transformants tested.

${ }^{b}$ A single papilla was formed by only one of the four transformants and was presumed to be a revertant.

preventing complementation of gcd2-1 by GCN3 (42). To test this hypothesis, we determined the nature of all three $\operatorname{gcd} 2$ mutations. Our interest in the sequence of these mutant alleles was further stimulated by our recent finding that GCD7 exhibits strong sequence similarity with GCN3 and to a lesser extent with GCD2 (4).

We mapped the three $G C D 2$ mutations by marker rescue of their temperature-sensitive phenotypes. Deletion derivatives of $G C D 2$ were generated by using suitable restriction sites and subcloned into the high-copy-number yeast plasmid YEp24 (Table 1). As expected, none of these gcd2 deletion alleles complemented the $\mathrm{Tsm}^{-}$phenotype of the three $\mathrm{gcd} 2$ mutations. If the position of the chromosomal $g c d 2$ mutation is contained in the wild-type GCD2 sequences remaining in the plasmid, then homologous recombination events between the chromosome and the plasmid can generate the wild-type allele of GCD2. We tested for such events in transformants of various $g c d 2$ mutants containing the plasmid-borne $g c d 2$ deletion alleles by observing the frequency of temperature-resistant (wild-type $G C D 2$ ) papillae that arose spontaneously in each transformant (Table 4). The results of this analysis localized the gcd2-502 and gcd2-503 mutations to the 343-bp interval between the Bam HI and NdeI sites near the middle of GCD2, whereas the gcd2-1 mutation was localized to the C-terminal segment $3^{\prime}$ of the NdeI site in GCD2.

We then used PCR to amplify and clone the appropriate regions containing the $g c d 2$ mutant alleles from genomic DNA and determined the nature of each mutation by DNA sequence analysis (see Materials and Methods). The gcd2502 and $\operatorname{gcd} 2-503$ mutations were found to contain singlepoint mutations resulting in glycine and serine substitutions, respectively, at the same amino acid, arginine 334 . At odds with the complementation model described above, this residue is located in a region of GCD2 that shows no significant sequence similarity with GCN3 or GCD7 (Fig. 2). Moreover, the gcd2-1 allele contains a nonsense mutation that eliminates the last 21 amino acids from the $C$ terminus of GCD2, thus affecting the region of highest similarity among these three proteins. The location of these gcd 2 mutations suggests that the ability of $G C N 3$ to completely suppress the lethal effects of $g c d 2-502$ and $g c d 2-503$, but not that of $\operatorname{gcd} 2-1$, cannot be explained simply by proposing that the regions of sequence similarity between GCD2 and GCN3 represent different copies of a functionally redundant domain that is impaired by $g c d 2-502$ and gcd2-503 but unaffected by $\operatorname{gcd} 2-1$. Rather, it appears that these mutations 


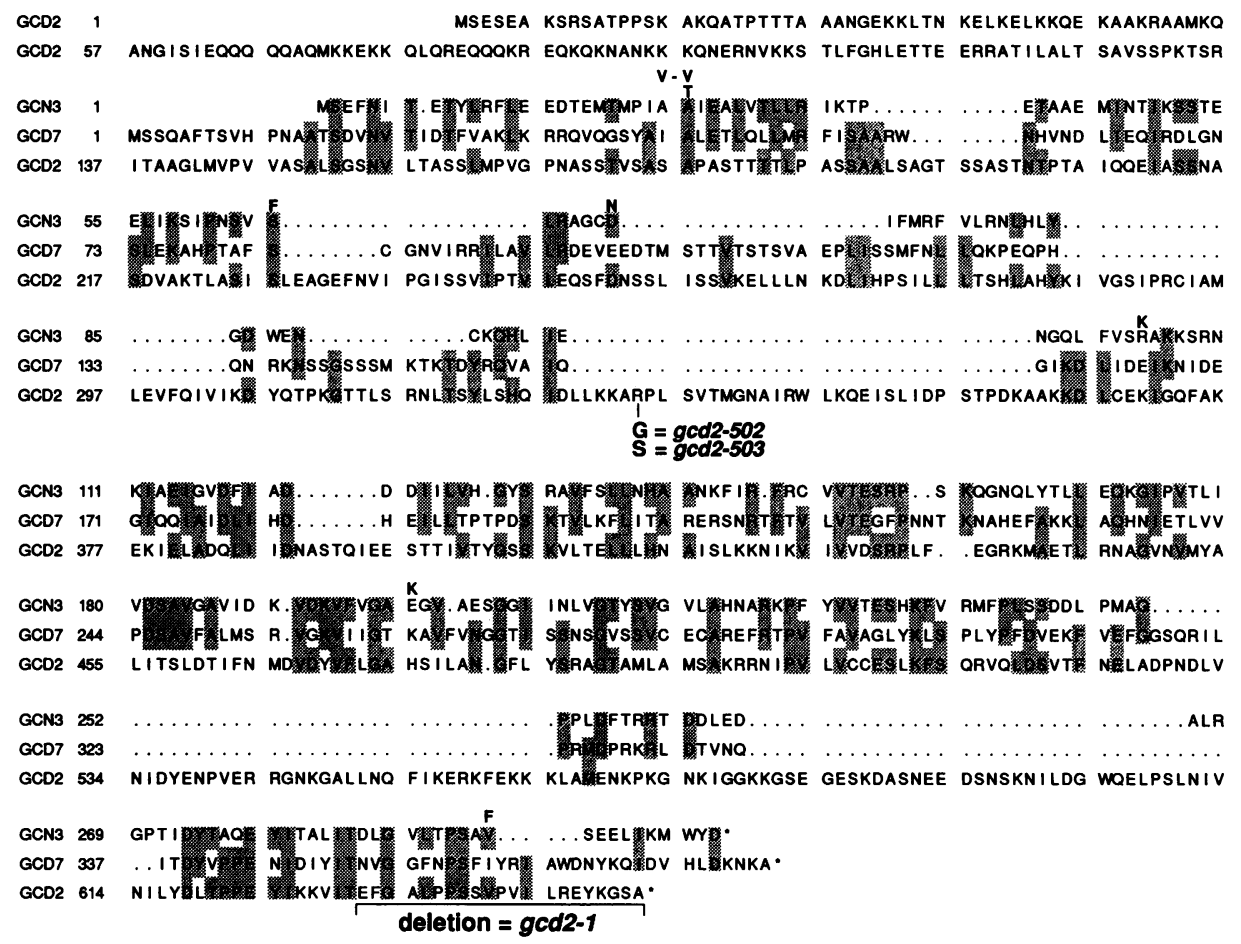

FIG. 2. Amino acid sequence changes caused by gcd 2 mutations. The amino acid sequences of GCN3, GCD7, and GCD2 were aligned as reported previously (4). Identities are shaded, and gaps in the alignments are indicated with dots. Amino acid changes resulting from gcn $3^{\mathrm{c}}$ mutations are shown above the GCN3 sequence. The changes resulting from gcd2 mutations are shown below the GCD2 sequence.

affect the function of the GCD-eIF-2B complex in ways that are differentially influenced by the presence of GCN3.

Allele-specific interactions between $\operatorname{gcn}^{c}$ mutations and different alleles of $G C D 2$. An alternative explanation for the sequence relatedness between GCN3 and GCD2 is that the regions of similarity mediate interactions between these two proteins in the GCD-eIF-2B complex. If this hypothesis is correct, it should be possible to detect extensive allele specificity in the interactions between mutations in GCD2 and $G C N 3$. We reasoned that the $g c n 3^{c}$ alleles isolated previously (17) provide a group of altered-function GCN3 mutations ideal for probing interactions between GCN3 and GCD2. As mentioned above, $g c n 3^{c}$ alleles resemble gcd2 mutations in causing constitutive derepression of GCN4 expression and slow growth under nonstarvation conditions; however, they do not confer temperature-sensitive growth. The $g c n 3^{c}$ mutations are located throughout the GCN3 sequence (Fig. 2), and their recessive nature suggests that they encode proteins which assemble into the GCD-eIF-2B complex less efficiently than does wild-type GCN3. Thus, if GCD2 and GCN3 are in physical contact, certain $g c n 3^{c}$ mutations might exhibit markedly different interactions with the $g c d 2-1$ allele that truncates the protein at the $\mathrm{C}$ terminus versus the $g c d 2-502$ and $g c d 2-503$ mutations that alter arginine 334 . To test this possibility, we constructed the full matrix of double mutants that combine $\operatorname{gcd} 2-502, \operatorname{gcd} 2-503$, or $g c d 2-1$ with each of seven different $g c n 3^{c}$ alleles and characterized the double mutants for the ability to form colonies at temperatures permissive $\left(23^{\circ} \mathrm{C}\right)$ or restrictive $\left(36^{\circ} \mathrm{C}\right)$ for $g c d 2$ single mutants (Table 5).

If GCN3 and GCD2 both contribute to the essential function of eIF-2B, we would expect that most $\operatorname{gcn} 3^{\mathrm{C}} \operatorname{gcd} 2$ double mutants would be more impaired for growth than is either single mutant. This type of additive interaction was observed for many of the double mutants, which displayed a growth defect at $23^{\circ} \mathrm{C}$ equal to or greater than that of the corresponding $\operatorname{gcn} 3^{\mathrm{c}}$ single mutant and showed poorer growth at $36^{\circ} \mathrm{C}$ than at $23^{\circ} \mathrm{C}$, characteristic of the temperature-sensitive $g c d 2$ mutations. This additivity was particularly striking in the case of gcd2-503, which was inviable in combination with four of the $g c n 3^{c}$ alleles, $g c n 3^{c}-A 26 T$, $g c n 3^{c}-A A 25,26, V V, g c n 3^{c}-E 199 K$, and $g c n 3^{c}-V 295 F$. Not surprisingly, this lethality involved the four $g c n 3^{c}$ alleles that conferred the slowest growth rate in the wild-type GCD2 strain. Three of these alleles $\left(g c n 3^{c}-A 26 T, g c n 3^{c}\right.$ $A A 25,26, V V$, and $\left.g c n 3^{c}-E 199 K\right)$ also produced the most growth-impaired $\operatorname{gcn} 3^{\mathrm{c}}$ gcd2-502 double mutants, one of which was inviable.

In contrast with the additive interactions just described, the slow growth rate at $23^{\circ} \mathrm{C}$ associated with several of the $g c n 3^{c}$ alleles was suppressed by certain mutations in $g c d 2$, and the temperature sensitivity of $g c d 2$ mutants was suppressed by certain $g c n 3^{c}$ alleles, in some cases even when the $g c n 3^{c}$ was very deleterious in the $G C D 2$ strain. The slow growth at $23^{\circ} \mathrm{C}$ conferred by $g c n 3^{c}-V 295 F$, gcn $3^{c}-R 104 K$, and $g c n 3^{c}-S 65 F$ was significantly suppressed by $g c d 2-502$ and $g c n 3^{\mathrm{c}}-\mathrm{S} 65 \mathrm{~F}$ was also suppressed by $g c d 2-503$. Another noteworthy feature of the $g c n 3^{\mathrm{c}}-\mathrm{R} 104 \mathrm{~K}$ and $g c n 3^{\mathrm{c}}-\mathrm{S} 65 \mathrm{~F}$ alleles is that they conferred better growth at $36^{\circ} \mathrm{C}$ in the $g c d 2-502$ and $g c d 2-503$ strains than did $g c n 3^{c}-D 71 N$, even though the latter allele produced a lesser growth defect at $23^{\circ} \mathrm{C}$ in the $G C D 2$ strain. These suppressive interactions suggest that the mutations involved cause offsetting biochemical defects, or that the GCN3 and GCD2 proteins are in close contact in eIF-2B and these mutations lead to offsetting structural changes in the complex.

There were many similarities in the interactions between the various $g c n 3^{c}$ mutations and the $\operatorname{gcd} 2-502$ and $g c d 2-503$ 
TABLE 5. Genetic interactions among $g c n 3^{c}$ and $g c d 2$ alleles

\begin{tabular}{|c|c|c|c|c|c|c|c|c|}
\hline \multirow{3}{*}{$\begin{array}{l}\text { Plasmid-borne } \\
G C N 3 \text { allele }\end{array}$} & \multicolumn{8}{|c|}{ Colony formation in transformants of strain ${ }^{a}$ : } \\
\hline & \multicolumn{2}{|c|}{ GCD2 gcn3s } & \multicolumn{2}{|c|}{ gcd2-502 gcn3山 } & \multicolumn{2}{|c|}{ gcd2-503 gcn3山 } & \multicolumn{2}{|c|}{ gcd2-1 gcn $3 \Delta$} \\
\hline & $23^{\circ} \mathrm{C}$ & $36^{\circ} \mathrm{C}$ & $23^{\circ} \mathrm{C}$ & $36^{\circ} \mathrm{C}$ & $23^{\circ} \mathrm{C}$ & $36^{\circ} \mathrm{C}$ & $23^{\circ} \mathrm{C}$ & $36^{\circ} \mathrm{C}$ \\
\hline $\begin{array}{l}\text { None (vector) } \\
G C N 3 \\
g c n 3^{c}-A 26 T \\
g c n 3^{c}-A A 25,26 V V \\
g c n 3^{c}-S 65 F \\
g c n 3^{c}-D 71 N \\
g c n 3^{c}-R 104 K \\
g c n 3^{c}-E 199 K \\
g c n 3^{c}-V 295 F\end{array}$ & $\begin{array}{l}++++ \\
++++ \\
+ \\
+1- \\
++ \\
+++ \\
++ \\
+ \\
+\end{array}$ & $\begin{array}{l}++++ \\
++++ \\
++ \\
+ \\
+++ \\
++ \\
++ \\
+1- \\
+1-\end{array}$ & $\begin{array}{l}++ \\
++++ \\
+^{+} \\
\text {Lethal } \\
+++^{+} \\
+++ \\
+++^{+} \\
+ \\
++^{+}\end{array}$ & $\begin{array}{l}- \\
++++ \\
+/- \\
\text { Lethal } \\
+ \\
+/- \\
+ \\
- \\
-\end{array}$ & $\begin{array}{l}+ \\
++++ \\
\text { Lethal } \\
\text { Lethal } \\
+++^{+} \\
+++ \\
+^{+} \\
\text {Lethal } \\
\text { Lethal }\end{array}$ & $\begin{array}{l}- \\
++++ \\
\text { Lethal } \\
\text { Lethal } \\
+ \\
- \\
+/- \\
\text { Lethal } \\
\text { Lethal }\end{array}$ & $\begin{array}{l}++++ \\
++++ \\
++ \\
++ \\
+++ \\
++^{+} \\
++^{+} \\
++++ \\
+\end{array}$ & $\begin{array}{l}+1- \\
++ \\
+ \\
+ \\
+1- \\
+1- \\
++ \\
+ \\
+\end{array}$ \\
\hline
\end{tabular}

${ }^{a}$ Allelles of $G C N 3$ borne on the low-copy-number plasmid YCp50 (17) were introduced into gcn3D yeast strains H1426 (GCD2), EY462 (gcd2-502), H652 (gcd2-503), and H954 (gcd2-1). Growth was scored for five independent transformants by streaking for single colonies on minimal medium and incubating colonies at 23 or $36^{\circ} \mathrm{C}$. Relative colony sizes were scored 3 to 6 days later. A minus sign indicates that no visible colonies were formed. A superscript plus indicates less growth than that signified by a normal plus.

alleles, which may reflect the fact that these two mutations alter the same amino acid in GCD2. In general, combinations with $g c d 2-503$ had phenotypes similar to those seen with gcd2-502, but more severe. Notable exceptions involved $g c n 3^{\mathrm{c}}-R 104 \mathrm{~K}$ and $g c n 3^{\mathrm{c}}-V 295 \mathrm{~F}$, which were suppressed by gcd2-502 at $23^{\circ} \mathrm{C}$ but exacerbated by $g c d 2-503$. Some differences in the interactions among gcd2-502 and gcd2-503 and $g c n 3^{c}$ alleles were not unexpected, since these two gcd 2 mutations have quite different effects on polysome profiles in vivo (12).

The gcd2-1 mutation, which deletes the $\mathrm{C}$ terminus of the protein, displayed a distinctly different pattern of interactions with the $g c n 3^{c}$ alleles. In several cases, $g c n 3^{c}$ mutations that were exacerbated by combination with $g c d 2-502$ or gcd2-503 were suppressed by combination with gcd2-1. Specifically, the gcn $3^{\mathrm{c}}-A A 25,26 V \mathrm{~V}$ and $g c n 3^{\mathrm{c}}-\mathrm{E} 199 \mathrm{~K}$ mutations, which were very deleterious in the GCD2 strain and lethal or very severe in combination with $\operatorname{gcd} 2-502$ and gcd2-503, were suppressed for their growth defects at $23^{\circ} \mathrm{C}$ by $\operatorname{gcd} 2-1$. Finally, it is interesting that the $g c n 3^{c}-A 26 T$ gcd2-1 and $g c n 3^{c}-V 295 F$ gcd2-1 double mutants exhibit no temperature sensitivity and grow at rates very similar to those of the corresponding $g \mathrm{cn} 3^{\mathrm{c}}$ single mutants, suggesting that the $g c d 2-1$ mutation is completely suppressed by these two gcn $3^{c}$ alleles. The numerous instances of nonadditivity and mutual suppression seen in the interactions between the $\operatorname{gcd} 2$ mutations and $g c n 3^{\mathrm{c}}$ alleles, plus the fact that $g c d 2-1$ shows a distinct and specific set of interactions versus $\operatorname{gcd} 2-502$ and $\operatorname{gcd} 2-503$, suggest to us that the GCD2 and GCN3 proteins are in close physical contact within the GCD complex.

Genetic evidence that GCD6 and GCD7 are subunits of the GCD-eIF-2B complex. In view of our recent findings that gcd6 and gcd7 mutations affect GCN4 translational control, that GCD6 and GCD1, and GCD7, GCD2, and GCN3, are related in sequence, and that GCD6 is highly similar to the largest subunit of rabbit eIF-2B (4), we decided to determine whether GCD6 and GCD7 are additional subunits of the GCD-eIF-2B complex. We reasoned that if this hypothesis was correct, it might be possible to observe genetic interactions between $G C D 6$ or $G C D 7$ and $G C N 3$ similar to those just described for $G C D 2$. To test our prediction, we constructed double mutants containing gcd6-1 or gcd7-201 and one of four different $G C N 3$ alleles: wild-type $G C N 3$, a gcn3 deletion, $g c n 3^{c}-R 104 K$, or gcn3-102.

As observed for a mutation in the GCD1 subunit of the
GCD-eIF-2B complex $(19,26)$, deletion of $G C N 3$ was lethal in the presence of $g c d 6-1$ and $g c d 7-201$ (Table 6). This result is striking because in an otherwise wild-type strain, deletion of GCN3 does not reduce cell viability and its effect on GCN4 expression is the opposite of that associated with the gcd6-1 and gcd7-201 mutations (see below). We also observed synthetic lethality between the gcd6-1 and gcd7-201 mutations and the $g c n 3^{c}-R 104 K$ allele. These interactions are consistent with the idea that GCD6 and GCD7 are subunits of the GCD-eIF-2B complex and that gcd6-1 and gcd7-201 impair these subunits in a way that is exacerbated either by eliminating GCN3 or by altering it in a way that reduces the biochemical function of the complex.

The gcn3-102 allele is defective for promoting derepression of GCN4 expression; however, it resembles wild-type GCN3 in its ability to suppress the temperature-sensitive growth exhibited by certain $g c d 1$ and $g c d 2$ mutations (20). Similar to its effect on gcd1 and gcd2 mutations, the gcn3102 allele did not exacerbate the growth defect of gcd6-1 and completely suppressed the slow-growth phenotype of gcd7-

TABLE 6. Genetic interactions between different alleles of GCN3 and the gcd6-1 and gcd7-201 alleles

\begin{tabular}{|c|c|c|c|}
\hline Relevant genotype $^{a}$ & Growth rate $^{b}$ & 3-AT ${ }^{\mathrm{Tc}}$ & $\begin{array}{c}\text { Effect of } G C N 3 \\
\text { mutant allele }\end{array}$ \\
\hline 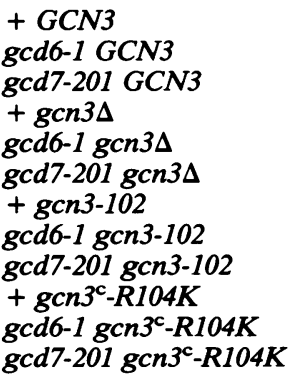 & $\begin{array}{l}+ \\
+/- \\
+1- \\
+ \\
\text { Lethal } \\
\text { Lethal } \\
+ \\
+1- \\
+ \\
+1- \\
\text { Lethal } \\
\text { Lethal }\end{array}$ & $\begin{array}{l}- \\
+ \\
+1- \\
+\end{array}$ & $\begin{array}{l}\text { Exacerbate } \\
\text { Exacerbate } \\
\text { None } \\
\text { Suppress } \\
\text { Exacerbate } \\
\text { Exacerbate }\end{array}$ \\
\hline
\end{tabular}

${ }^{a}$ Double-mutant combinations were generated from crosses as described in Materials and Methods. +, wild-type alleles for GCD6 and GCD7.

${ }^{b}$ Scored relative to the wild-type rate $(+)$ by colony size after 1 to 2 days at $30^{\circ} \mathrm{C}$.

${ }^{c}$ Scored by replica plating to SD medium supplemented with 3-AT and scoring growth relative to the wild-type level $(+)$ after 2 to 3 days at $30^{\circ} \mathrm{C}$. The level of 3-AT resistance is an indicator of the induced levels of GCN4 expression $\left(+, 3-\mathrm{AT}^{\mathrm{T}}\right.$, high $G C N 4$ expression;,$- 3-\mathrm{AT}^{\mathrm{s}}$, low and uninducible GCN4 expression) 
TABLE 7. Effects of $g c n 3-102$ and $g c d 7-201$ mutations on GCN4 expression

\begin{tabular}{lclccc}
\hline Strain & $\begin{array}{c}\text { Ascospore } \\
\text { clone }^{a}\end{array}$ & Relevant genotype & \multicolumn{2}{c}{$G C N 4$-lacZ expression } \\
\cline { 4 - 5 } & & Nonstarvation & $\begin{array}{c}\text { Histidine } \\
\text { starvation }\end{array}$ \\
\hline H1875 & 1C & GCN3 GCD7 & 10 & 73 \\
H1882 & 2D & GCN3 GCD7 & 17 & 76 \\
H1876 & 1D & GCN3 gcd7-201 & 190 & 170 \\
H1880 & 2C & GCN3 gcd7-201 & 170 & 140 \\
H1872 & 1A & gcn3-102 GCD7 & 7 & 15 \\
H1879 & 2B & gcn3-102 GCD7 & 11 & 24 \\
H1873 & 1B & gcn3-102 gcd7-201 & 23 & 57 \\
H1877 & 2A & gcn3-102 gcd7-201 & 23 & 83 \\
\hline
\end{tabular}

${ }^{a}$ The low-copy-number plasmid p180 bearing GCN4-lacZ was introduced into ascospore clones $1 \mathrm{~A}$ to $1 \mathrm{D}$ and $2 \mathrm{~A}$ to $2 \mathrm{D}$ from two tetratype tetrads ( 1 and 2) from the cross between H1603 (gcd7-201 GCN3) and H1839 (GCD7::URA3 gcn3-102); see Materials and Methods for details.

${ }^{b} \beta$-Galactosidase activity in yeast strains grown to mid-logarithmic phase under repressing (nonstarvation) conditions or under derepressing conditions of histidine starvation induced by 3-AT was measured as described previously (35). Enzyme activities are expressed as units (nanomoles of $o$-nitrophenyl$\beta$-D-galactopyranoside [ONPG] cleaved per minute per milligram of total protein). Results are mean values of measurements made on two independent transformants for each strain; individual measurements differed from the mean by less than $20 \%$.

201 (Table 6). It is noteworthy that gcn3-102 was isolated as a suppressor of the temperature-sensitive phenotype of gcd1-101 in a GCN3 strain (26). These results can be explained by proposing that the gcn3-102 product is competent for complex formation and that it compensates for defects in the structure or function of the GCD-eIF-2B complex associated with particular mutations in GCD1 or GCD7.

The inability of gcn3-102 to promote derepression of GCN4 expression is suppressed by the gcd6-1 mutation, as indicated by the wild-type resistance to 3-AT exhibited by the double mutant (Table 6). 3-AT inhibits histidine biosynthesis, and wild-type resistance requires efficient derepression of genes in the histidine pathway subject to transcriptional regulation by GCN4. In contrast to what was seen with gcd6-1, the 3-AT ${ }^{\mathrm{s}}$ phenotype of gcn3-102 was not fully suppressed by gcd7-201 (Table 6). To quantitate the effects on GCN4 expression associated with combining these last two mutations, we assayed the expression of a GCN4-lacZ fusion in strains containing different combinations of the gcd7-201 and gcn3-102 mutant alleles (Table 7).

The wild-type GCN3 GCD7 strain showed the expected increase in GCN4-lacZ expression under conditions of histidine starvation. The gcn3-102 single mutant was impaired for this derepression, consistent with the idea that GCN3 is the regulatory subunit of eIF-2B that couples increased translation of $G C N 4$ to reduced amino acid availability. The gcd7-201 single mutant showed constitutive derepression of GCN4-lacZ expression, as would be expected for a reduction in eIF-2B function irrespective of amino acid availability. The double mutant gave GCN4-lacZ expression intermediate between that seen in the single mutants and similar to what we observed in the wild-type strain. Thus, the gcd7-201 and gcn3-102 mutations appear to be compensatory in nature.

Coimmunoprecipitation of GCD6 and GCD7 with other subunits of the GCD-eIF-2B - eIF-2 complex. The genetic interactions between mutations in GCN3 and the gcd6-1 and gcd7-201 alleles described above lent strong support to the idea that GCD6 and GCD7 are present with GCN3 in the

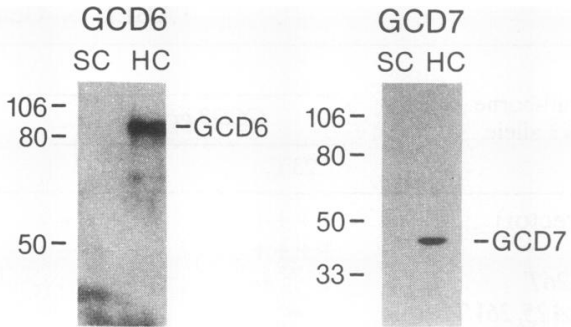

FIG. 3. Characterization of GCD6-specific and GCD7-specific antibodies by immunoblot analysis of unfractionated yeast extracts. For GCD6, 50- $\mu \mathrm{g}$ samples of extracts prepared from wild-type strain H1730 bearing vector pRS425 (SC) or high-copy-number GCD6 plasmid pJB115 (HC) were fractionated by SDS-PAGE (10\% polyacrylamide gel) and subjected to immunoblot analysis using a 1:500 dilution of GCD6-specific antiserum. The immunoreactive species visible only in the HC extract is presumed to be GCD6 and is labeled as such. For GCD7, 50- $\mu \mathrm{g}$ samples of extracts prepared from wild-type strain $\mathrm{H} 1727$ bearing vector alone (SC) or high-copynumber $G C D 7$ plasmid pJB111 (HC) were analyzed as for GCD6, using a 1:250 dilution of GCD7-specific antiserum. The immunoreactive species visible only in the HC extract is presumed to be GCD7 and is labeled as such. Migration of Bio-Rad prestained low-molecular-weight markers is indicated on the left of each panel, with the molecular weights of the markers given in kilodaltons.

GCD-eIF-2B complex. In an effort to obtain physical evidence for this interaction, we used antibodies against GCD6 and GCD7 to investigate whether they coimmunoprecipitate and copurify with other subunits of the GCD-eIF-2B - eIF-2 complex. The antibodies were raised against TrpE-GCD6 and TrpE-GCD7 fusion proteins produced in E. coli (see Materials and Methods), and their specificity was established by immunoblot analysis of total protein extracts of wild-type strains and transformants containing high-copynumber plasmids bearing $G C D 6$ or GCD7. As shown in Fig. 3 , each antiserum reacted with a single polypeptide having an electrophoretic mobility consistent with the predicted molecular weight of the corresponding GCD protein $(81,000$ for GCD6 and 43,000 for GCD7 [4]) that was present at higher levels in the transformants containing the appropriate high-copy-number plasmids.

The GCD6-specific antiserum was used to immunoprecipitate proteins under nondenaturing conditions from a highsalt extract of yeast ribosomes (RSW) found previously to be enriched for the GCD1, GCD2, and GCN3 proteins (6). The immunoprecipitated proteins were fractionated by sodium dodecyl sulfate-polyacrylamide gel electrophoresis (SDSPAGE) and analyzed by immunoblotting with antibodies against GCD6, GCD7, GCD1, GCD2, GCN3, and the $\alpha$ and $\beta$ subunits of eIF-2 (Fig. 4). We found that GCD6, GCD7, GCD2, GCN3, and GCD1 proteins present in the RSW were recovered in the immune complexes (Fig. 4A, lanes 2 and 7) and substantially depleted from the supernatants (Fig. 4B, lanes 2 and 7). In addition, a small fraction of the eIF-2 $\alpha$ and eIF-2 $\beta$ subunits were coimmunoprecipitated with GCD6. In control immunoprecipitations done with preimmune serum, none of these proteins was isolated in the immune complexes or depleted from the supernatants (Fig. 4A and B, lanes 3 and 8 ). The depletion of proteins from the supernatants by immunoprecipitations using GCD6-specific antibodies indicates that the majority of the GCD7, GCD2, GCN3, and GCD1 proteins in the RSW are complexed with GCD6.

These results suggest that GCD6 and GCD7 are present in the same complexes that were previously found to contain GCD1, GCD2, GCN3, and a fraction of eIF-2 (6). If this is 


\section{Immunoprecipitates}

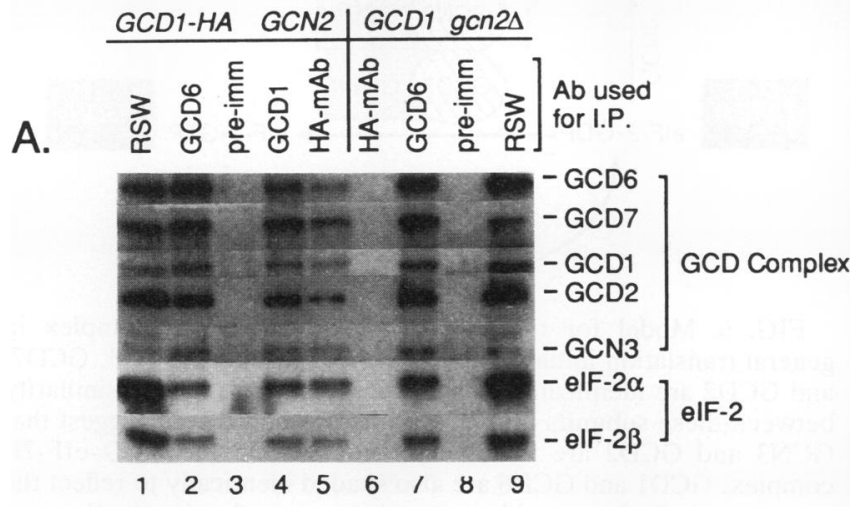

Supernatants

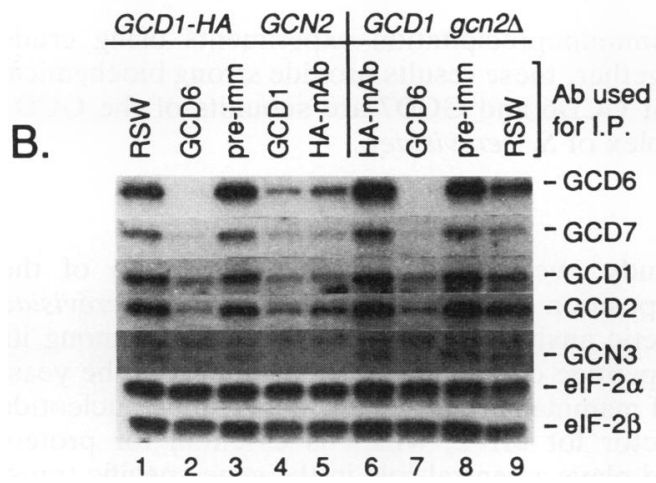

FIG. 4. Coimmunoprecipitation of GCD6 and GCD7 with subunits of the GCD-eIF-2B - eIF-2 complex. Proteins were immunoprecipitated from RSW extracts prepared from strain MC1057 (GCD1-HA GCN2) or H1333 (wild-type GCD1 gcn2A), as indicated. Lanes 1 and 9 (RSW) contain $47 \mu \mathrm{g}$ of the extract used for the immunoprecipitations in panel A and $19 \mu \mathrm{g}$ in panel B; all other lanes in panel A contain immunoprecipitated material from $52 \mu \mathrm{g}$ of the RSW extract; all other lanes in panel B contain a portion of the supernatants remaining after immunoprecipitation corresponding to $24 \mu \mathrm{g}$ of the RSW extract. Antibodies used for immunoprecipitation (Ab used for I.P.) are indicated above lanes 2 to 8 . pre-imm, preimmune antiserum from the rabbit in which the GCD6-specific antibody was raised; HA-mAb, the monoclonal antibody that recognizes the HA epitope used to epitope tag GCD1 in strain MC1057. Samples of the immunoprecipitates and supernatants were fractionated by SDS-PAGE (10\% polyacrylamide gel) and subjected to immunoblot analysis with the antibodies indicated on the right to visualize the different subunits of the GCD-eIF-2B and eIF-2 complexes. For the immunoblot analysis, several identical blots were prepared for each set of samples and divided across the lanes into strips containing the appropriate molecular weight fractions for each antigen being detected. The identities of the immunoreactive species have been established rigorously in previous studies (6) for GCD1, GCD2, GCN3, eIF-2 $\alpha$, and eIF-2 $\beta$ and in this study for GCD6 and GCD7 (Fig. 3). A cross-reacting band migrating slightly more slowly than GCN3 was detected with the GCN3 antibody in the RSW but not in the immunoprecipitates. This band was present at the same levels in the RSW and the supernatant fractions. More than $95 \%$ of the nonspecific proteins remained in the supernatants, as judged by Ponceau S staining of the nitrocellulose filters prior to immunoblot analysis. true, we would expect to find that GCD6 and GCD7 can be quantitatively immunoprecipitated by an antiserum against one of the other subunits of the complex. In accord with this expectation, we obtained results essentially identical to those just described for GCD6-specific antiserum when we used antibodies against GCD1 instead (compare lanes 2 and 4 in Fig. 4A and B). As a final control, we showed that GCD6 and GCD7 could be immunoprecipitated by a monoclonal antibody that recognizes an epitope-tagged version of GCD1. An allele of GCD1 in which nine amino acids corresponding to an epitope of the hemagglutinin (HA) protein of influenza virus were added to the $C$ terminus of $G C D 1$ has been constructed (5). This epitope-tagged version of GCD1 complements a gcdl deletion like the wild-type allele, and the HA-tagged GCD1 protein can be immunoprecipitated by a monoclonal antibody against the HA epitope (5). As shown in Fig. 4, this monoclonal antibody coimmunoprecipitated a significant portion of the GCD6, GCD7, and GCD2 proteins and a small fraction of eIF- $2 \alpha$ and eIF- $2 \beta$ along with the majority of the GCD1 and GCN3 present in the RSW prepared from yeast cells expressing the epitope-tagged version of GCD1. In contrast, none of these proteins was immunoprecipitated by the monoclonal antibody when the RSW was prepared from a strain expressing wild-type $G C D 1$ (compare lanes 5 and 6 in Fig. 4A and B). The GCD6 and GCD2 proteins were selectively depleted from these immunoprecipitates compared with those obtained by using antibodies against GCD1 or GCD6. We presume that either addition of the HA epitope or binding of the HA monoclonal antibody to GCD1 partially disrupted the GCD-eIF-2B complex and decreased the affinity of GCD6 and GCD2 for the remainder of the complex.

By comparing immunoprecipitates from wild-type and $g c n 2 \Delta$ strains, we tested whether eIF- $2 \alpha$ phosphorylation was required for the association of a fraction of eIF- 2 with the GCD complex described above. In the case of factors purified from Ehrlich ascites tumor cells, eIF-2 phosphorylated on the $\alpha$ subunit was deduced to have an affinity 150-fold higher than that of the nonphosphorylated species for eIF-2B in vitro. This difference in affinity is believed to be important for the inhibition of eIF-2B function by phosphorylated eIF-2 (47). However, we found that the same fraction of eIF-2 subunits was coimmunoprecipitated by antibodies against GCD6 from an RSW prepared from a wild-type GCN2 strain or a gcn2 deletion strain (compare lanes 2 and 7 in Fig. 4A and B). As no eIF-2 $\alpha$ phosphorylation has been detected in a gcn2 deletion strain (10), we conclude that phosphorylation of eIF- $2 \alpha$ is not required for the interaction between the GCD-eIF-2B complex and eIF-2 detected under our immunoprecipitation conditions.

GCD6 and GCD7 copurify with other subunits of the GCD-eIF-2B - eIF-2 complex. To provide independent biochemical evidence that GCD6 and GCD7 are components of the GCD-eIF-2B complex, we used immunoblot analysis to determine whether GCD6 and GCD7 copurify with other subunits of the complex. Cigan et al. have recently described a purification scheme for the GCD-eIF-2B complex and shown that the most highly purified preparations of this complex contain the guanine nucleotide exchange activity for eIF-2 that is characteristic of mammalian eIF-2B (5). Typical results from one of the final steps in this purification scheme, velocity sedimentation on a glycerol gradient, are shown in Fig. 5. Immunoblot analysis of the gradient fractions revealed that GCD1, GCD2, and GCN3 were distributed between fractions 12 and 13 with most of the eIF- $2 \alpha$ and eIF- $2 \beta$ that were present in the starting sample. In similar 

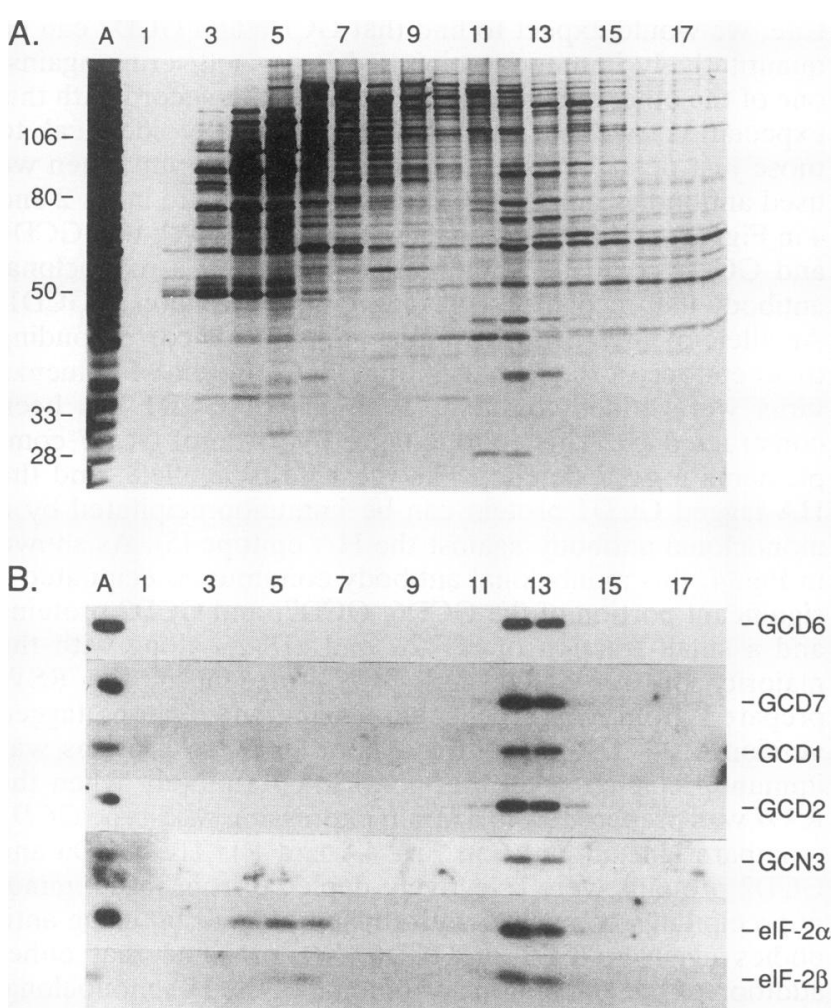

FIG. 5. Copurification of GCD6 and GCD7 with the GCDeIF-2B complex and subunits of eIF-2. Silver stain and immunoblot analyses of fractions from a glycerol gradient following velocity sedimentation of a protein fraction enriched for the GCD-eIF-2B complex are shown in panels $A$ and $B$, respectively. The $0.5 \mathrm{M} \mathrm{KCl}$ postribosomal supernatant obtained from a crude extract of yeast strain MC1057 was purified through four different ion-exchange chromatography steps to enrich for the GCD1 and GCD2 proteins as monitored by immunoblot analysis (5). This material was subjected to velocity sedimentation on a $\mathbf{1 5}$ to $30 \%$ glycerol gradient, and the resulting gradient fractions were analyzed by SDS-PAGE $(10 \%$ polyacrylamide gel), all as described previously (5). (A) Total proteins were visualized by silver staining. The positions and sizes (in kilodaltons) of molecular weight markers are indicated on the left. (B) Immunoblot analysis was used to detect the proteins listed on the right, using the antibodies described in the legend to Fig. 4. In both panels, lane A contains the material applied to the gradient; the gradient fractions are analyzed in lanes 1 to 18 , with the material from the top of the gradient in lane 1.

experiments, the peak guanine nucleotide exchange activity for eIF-2 was found in the same fractions containing the majority of these five proteins (5). Immunoblot analysis of the gradient fractions by using our antibodies against GCD6 and GCD7 showed that these proteins were present in the same two peak fractions containing the other subunits of the GCD-eIF-2B complex. The fact that GCD6 and GCD7 are major constituents of a protein fraction purified solely on the basis of the presence of GCD1 and GCD2 provides strong evidence that GCD6 and GCD7 are physically associated with the GCD1 and GCD2 proteins. In addition, Cigan et al. (5) have recently found that GCD6 and GCD7 can be immunopurified with six other subunits of the GCD-eIF2B - eIF-2 complex, starting with highly purified material from a strain expressing the HA epitope-tagged version of GCD1, using the monoclonal antibodies against the HA epitope. In the previous section, we reported similar conclu-

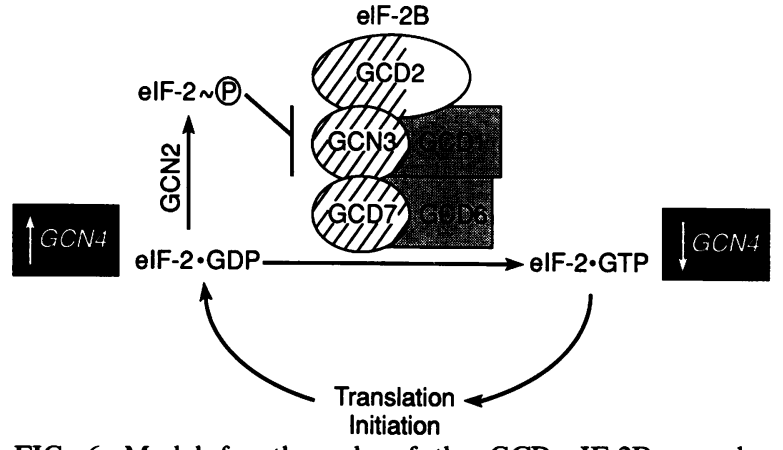

FIG. 6. Model for the role of the GCD-eIF-2B complex in general translation initiation and GCN4 expression. GCN3, GCD7, and GCD2 are identically shaded to reflect the sequence similarity between these subunits $(4,42)$. Results presented here suggest that GCN3 and GCD2 are in direct contact within the GCD-eIF-2B complex. GCD1 and GCD6 are also shaded identically to reflect the sequence similarity noted between these two subunits (4). See text for further details.

sions from immunoprecipitation experiments using crude extracts. Together, these results provide strong biochemical evidence that GCD6 and GCD7 are subunits of the GCDeIF-2B complex of $S$. cerevisiae.

\section{DISCUSSION}

In this study, we extend our characterization of the subunit composition of the GCD complex of $S$. cerevisiae and use genetic analysis to explore interactions among its various polypeptide components. This complex is the yeast equivalent of mammalian eIF-2B (5), the guanine nucleotide exchange factor for eIF-2, which is essential for protein synthesis and plays a central role in the gene-specific translational control of GCN4. Consistent with the view that the regulatory subunit GCN3 is an integral component of the GCD-eIF-2B complex, our observations suggest that $\operatorname{gcn} 3^{\mathrm{c}}$ mutations lead to defects in general translation initiation. Our genetic data showing extensive allele-specific interactions between $G C N 3$ and $G C D 2$ suggest that GCN3 and GCD2 interact directly within the GCD-eIF-2B complex. In addition, we obtained strong genetic and biochemical evidence that GCD6 and GCD7 are additional components of this complex (5). Mammalian eIF-2B contains five subunits (44) with molecular weights very similar to those deduced from the cloned genes encoding GCD1, GCD2, GCD6, GCD7, and GCN3, leading us to suggest that all of the subunits of eIF-2B in $S$. cerevisiae have now been identified $(4,5)$.

Figure 6 summarizes many of our conclusions about the structure and function of the GCD-eIF-2B complex. Its principal activity is guanine nucleotide exchange on eIF-2. Under normal growth conditions in wild-type cells, eIF2 . GTP is rapidly regenerated from eIF-2 . GDP and GTP, leading to high levels of translation initiation and repression of $G C N 4$. In response to amino acid starvation, the $\alpha$ subunit of eIF-2 is phosphorylated by the protein kinase GCN2. By analogy with mammalian systems, phosphorylated eIF-2 is thought to inhibit the GCD-eIF-2B complex. The resulting decrease in the levels of eIF-2 . GTP reduces the efficiency of general translation initiation and thereby stimulates the expression of GCN4. Mutations in one of the subunits of eIF-2B or eIF-2 itself can mimic the effect of eIF-2 phosphorylation in reducing the level of eIF-2 activity, with the 
attendant decrease in growth rate and derepression of GCN4.

Genetic properties of $G C N 3$ suggest a key regulatory role for this subunit of the GCD-eIF-2B complex. Deletion of GCN3 prevents translational derepression of GCN4 and overcomes the slow-growth phenotype associated with constitutive activation of GCN2 but does not affect cell growth or general translation under nonstarvation conditions (19, 25). Furthermore, recent results (9) indicate that deletion of $G C N 3$ does not diminish the phosphorylation of eIF- $2 \alpha$ by GCN2. These findings have led to the idea that GCN3 directly interacts with the $\alpha$ subunit of eIF-2 and mediates the inhibition of eIF-2B function exerted by the phosphorylated form of eIF-2 $(5,9)$.

Evidence that GCD6 and GCD7 are subunits of the GCD complex. Allele-specific interactions between GCD6 and $G C D 7$ and alleles of $G C N 3$ provided strong genetic evidence that GCD6 and GCD7 are components of the eIF-2B - eIF-2 complex of $S$. cerevisiae. The synthetic lethality reported here between mutations in GCD6 or GCD7 and a deletion of $G C N 3$ is in accord with earlier observations that certain mutations affecting GCD1 and GCD2 and the $\alpha$ and $\gamma$ subunits of eIF-2 (encoded by $S U I 2$ [7] and GCD11 [18], respectively) were more severe in strains lacking $G C N 3$ than in otherwise wild-type strains $(19,20,53)$. By showing that GCD6 and GCD7 coimmunoprecipitate and copurify with the GCD1, GCD2, and GCN3 subunits of the GCD-eIF-2B complex and with a fraction of the $\alpha$ and $\beta$ subunits of eIF-2, we provided two independent lines of biochemical evidence establishing that GCD6 and GCD7 are subunits of eIF-2B. Thus, six of the eight different polypeptides contained in the GCD-eIF-2B - eIF-2 complex of $S$. cerevisiae, GCD1, GCD2, GCD6, GCD7, GCN3 (subunits of eIF-2B), and GCD11 (the $\gamma$ subunit of eIF-2), were first identified genetically as translational regulators of $G C N 4$ expression. This finding provides strong support for the idea that translational derepression of $G C N 4$ expression is mediated by a reduction in the recycling of eIF-2 catalyzed by eIF-2B (Fig. 6).

Involvement of GCN3 in the essential function of the GCDeIF-2B complex in translation initiation. Although GCN3 is a dispensable subunit of yeast eIF-2B and is thought to be required primarily for down-regulation of eIF-2B activity by phosphorylated eIF-2 $\alpha$ (29), it can be mutated to have strong inhibitory effects on cell growth in the absence of starvation. We showed that $g c n 3^{c}$ mutations with this phenotype lead to reduced polysome content and increased amounts of $80 \mathrm{~S}$ monosomes relative to wild-type yeast strains. This polysome defect is characteristic of a reduction in the initiation stage of protein synthesis with little or no accompanying defect in elongation or termination. Thus, with initiation occurring slowly but elongation and termination proceeding at normal rates, the number of translating $80 \mathrm{~S}$ ribosomes on polysomes at any given time is diminished from the wildtype state. Under the conditions of our sucrose gradient analysis, the excess $40 \mathrm{~S}$ and $60 \mathrm{~S}$ subunits generated by polysome runoff accumulate as nonfunctional $80 \mathrm{~S}$ couples (12).

A second indication that general translation initiation is impaired in $g c n 3^{c}$ mutants came from our finding that two of the most severely affected $g c n 3^{c}$ alleles produce an accumulation of eIF-2 $\beta$ in the region of the polysome gradient containing small ribosomal subunits. This could indicate an accumulation of $48 \mathrm{~S}$ preinitiation complexes containing eIF2 . GTP - Met-tRNA ${ }_{i}^{\text {Met }}$ mRNA, and other initiation factors bound to $40 \mathrm{~S}$ subunits in $g c n 3^{c}$ strains. The same phenomenon has been observed in gcd1-101 (6) and gcd2-503 (12) mutants and in mammalian cell lysates in which eIF-2B function was inhibited $(8,13,14,52)$ and may be an indication that eIF-2B functions late in the initiation pathway at the stage of subunit joining. This hypothesis would explain the occurrence of halfmer polysomes in gcd-502 mutants (12) as an accumulation of $48 \mathrm{~S}$ preinitiation complexes on mRNAs containing translating $80 \mathrm{~S}$ ribosomes. It is interesting that the gcd2-502 and gcd2-503 mutations have markedly different effects on polysome profiles despite the fact that both mutations alter the same amino acid in GCD2. Perhaps these substitutions have differential effects on two distinct functions of eIF-2B in the initiation pathway.

Interactions between GCN3 and other subunits of the GCDeIF-2B complex. A common feature of most gcd mutations affecting the essential subunits of eIF-2B is that deletion of $G C N 3$ exacerbates the slow-growth phenotype associated with the mutation. One way to account for this interaction has been to propose that the presence of GCN3 in the GCD complex lessens the destabilizing effect of the gcd mutation on the structure or function of the complex (42). The postulated reduction in eIF-2B activity resulting from inactivation of $G C N 3$ would not be severe enough to reduce the rate of initiation in otherwise wild-type cells but would have noticeable consequences when combined with defects in other eIF-2B subunits, including the gcd6-1 and gcd7-201 mutations analyzed here. This additivity of separate biochemical defects can also explain how mutations in the $\alpha$ and $\gamma$ subunits of eIF- 2 can be exacerbated by deletion of $G C N 3$ $(20,53)$, as GCN3 is a subunit of the eIF-2-recycling factor.

The gcn3-102 mutation is interesting because it resembles a $g c n 3$ deletion in preventing derepression of $G C N 4$ under starvation conditions (28), suggesting that it renders the GCD-eIF-2B complex insensitive to phosphorylated eIF-2. It resembles wild-type $G C N 3$, however, in not exacerbating the effects of gcd mutations, suggesting that the gcn3-102 protein is stably incorporated into the GCD-eIF-2B complex and does not appreciably decrease the efficiency of guanine nucleotide exchange on nonphosphorylated eIF-2. The gcn3102 product is superior to wild-type $G C N 3$ for suppressing the gcd1-101 and gcd7-201 mutations but not for suppressing other gcd alleles, such as gcd6-1. This observation implies that the $g c d 1-101$ and $g c d 7-201$ mutations decrease eIF-2B activity by a common mechanism that differs from that involved with mutations like gcd6-1 which are not suppressed by $g c n 3-102$. We suspect that the $g c n 3-102$ product increases the activity of the GCD-eIF-2B complex in a way that offsets the particular biochemical defect(s) associated with the gcd1-101 and gcd7-201 mutations.

A panel of seven $g c n 3^{c}$ alleles was found to have substantially different patterns of interaction with the gcd2-502, gcd2-503, and $g c d 2-1$ mutations, and we observed several striking cases of mutual suppression by particular $g c n 3^{\mathbf{c}}$ and $\operatorname{gcd} 2$ alleles. First, we consider the suppression of $g c d 2$ mutations by $g c n 3^{c}$ alleles. Because wild-type $G C N 3$ either partially or completely suppresses the growth defects of the gcd 2 mutations, one component of the suppression conferred by the $g c n 3^{c}$ alleles probably involves the residual wild-type function of the $g c n 3^{c}$ product. However, the $g c n 3^{c}$ alleles displayed distinctly different profiles of suppression of the three gcd 2 mutations at $36^{\circ} \mathrm{C}$. For example, gcn $3^{c}$ $E 199 K$ and $g c n 3^{c}-V 295 F$ gave no suppression of $g c d 2-502$, exacerbated $g c d 2-503$, and suppressed $g c d 2-1$ nearly as well as did wild-type $G C N 3$. In addition, $g c n 3^{c}-D 71 N$ and $g c n 3^{c}$ $S 65 F$ gave no suppression of $g c d 2-1$ but were two of the best suppressors of $g c d 2-502$ and $g c d 2-503$. These results imply that different $g c n 3^{c}$ alleles and the three $\operatorname{gcd} 2$ mutations have 
distinguishable effects on the structure and function of the eIF-2B complex. A particular gcn $3^{c}$ product is able to compensate for one gcd 2 lesion but not another, and it may contribute an additional defect that further impairs the structure or function of the complex.

Of greater interest were the cases in which a gcd 2 mutant allele was superior to wild-type GCD2 in suppressing the growth defect of certain $g c n 3^{\mathrm{c}}$ mutations at $23^{\circ} \mathrm{C}$. These interactions indicate that a mutant gcd 2 protein can compensate for a defect caused by a particular $g c n 3^{\mathrm{c}}$ mutation that exhibits a similar phenotype. The most striking examples of this phenomenon were the suppression of $g c n 3^{\mathrm{c}}-A 26 \mathrm{~T}$ and gcn $3^{\mathrm{c}}-E 199 \mathrm{~K}$ by $g c d 2-1$ and the suppression of $g c n 3^{\mathrm{c}}-\mathrm{R} 104 \mathrm{~K}$ and $g c n 3^{c}-V 295 F$ by $g c d 2-502$. One way to account for this type of interaction would be to suggest that the two mutant proteins have offsetting biochemical defects. For example, a $g c n 3^{c}$ mutation might lead to an excessively stable interaction between eIF-2 and eIF-2B, mimicking the inhibitory effect of eIF-2 $\alpha$ phosphorylation on the recycling function of eIF-2B. A $g c d 2$ mutation that reduces eIF-2B-recycling function by weakening the interaction between eIF-2B and its substrate eIF- 2 could suppress this particular $g c n 3^{\mathrm{c}}$ allele.

Although we cannot rule out the possibility that all of the cases of mutual suppression that we observed are the result of compensatory biochemical defects, we believe that at least some of these interactions are indicative of direct contact between GCD2 and GCN3 in the eIF-2B complex. This view is motivated by our observation of regions of sequence similarity between these two proteins, which could form a conserved structural motif that mediates proteinprotein interactions. This notion, combined with the fact that regions of sequence similarity between GCN3 and GCD2 are also conserved in GCD7 (Fig. 2), leads to the idea that these three proteins form a heterotrimeric unit within the GCDeIF-2B complex that is stabilized by interactions between conserved regions of each protein (Fig. 6). Given that GCN3 is a nonessential subunit of the complex that seems to be required only for the regulation of eIF-2B function by phosphorylated eIF-2, perhaps GCD7 and GCD2 also have important regulatory roles, whereas the GCD1 and GCD6 proteins might be concerned primarily with catalysis.

Allele-specific interactions characteristic of multiprotein complexes. The observation of both synthetic lethality and mutual suppression between alleles of two genes is a strong indication that the factors involved form a multiprotein complex, as shown here for eIF-2B - eIF-2 of $S$. cerevisiae. The interactions that we observed among the trans-acting regulators of $G C N 4$ are remarkably similar to allele-specific interactions seen among other groups of genes, including: (i) interactions between a translational activator of mitochondrial genes and mitochondrial ribosomal proteins $(15,16$, 37), (ii) $c d c$ genes implicated in initiation of DNA replication in $S$. cerevisiae (22), (iii) certain transcription factors postulated to function as intermediaries between transcriptional activators and the basic transcription machinery $(46,51)$, and (iv) actin and $S A C$ genes presumed to encode other cytoskeletal components $(2,41)$. The high frequency with which we observed synthetic lethality among mutations in GCN3 and genes encoding subunits of eIF-2B or eIF-2 suggests that screens to identify synthetic lethal mutations may be extremely productive in the identification of interacting components. In addition, they provide a convenient way of cloning the genes once identified. While allelespecific interactions often have been interpreted as evidence for complex formation among a set of factors, in this study we attempted to use such genetic data to provide information about physical relationships among the components of the complex. This kind of detailed genetic approach to subunit interactions can provide a focus for molecular and biochemical studies. Thus, experiments are now under way to test the idea that cases of mutual suppression observed between different alleles of $G C N 3, G C D 2$, and $G C D 7$ arise from direct protein-protein interactions between these two subunits of the GCD-eIF-2B complex. In addition, it will be interesting to determine whether $\operatorname{gcd} 2-1$ and $g c n 3^{\mathrm{c}}-\mathrm{E} 199 \mathrm{~K}$ have offsetting effects on the eIF-2-recycling function of eIF-2B.

\section{ACKNOWLEDGMENTS}

We thank F. D. Bushman and C. Moehle for helpful comments on the manuscript.

\section{REFERENCES}

1. Abastado, J.-P., P. F. Miller, B. M. Jackson, and A. G. Hinnebusch. 1991. Suppression of ribosomal reinitiation at upstream open reading frames in amino acid-starved cells forms the basis for GCN4 translational control. Mol. Cell. Biol. 11:486-496.

2. Adams, A. E. M., and D. Botstein. 1989. Dominant suppressors of yeast actin mutations that are reciprocally suppressed. Genetics 121:675-683.

3. Boeke, J. D., J. Trueheart, G. Natsoulis, and G. R. Fink. 1987. 5-Fluoroorotic acid as a selective agent in yeast molecular genetics. Methods Enzymol. 154:164-175.

4. Bushman, J. L., A. I. Asuru, R. L. Matts, and A. G. Hinnebusch. 1993. Evidence that GCD6 and GCD7, translational regulators of $G C N 4$, are subunits of the guanine nucleotide exchange factor for eIF-2 in Saccharomyces cerevisiae. Mol. Cell. Biol. 13:1920-1932.

5. Cigan, A. M., J. L. Bushman, T. R. Boal, and A. G. Hinnebusch. A protein complex composed of translational regulators of GCN4 is the guanine nucleotide exchange factor for eIF-2 in yeast. Proc. Natl. Acad. Sci. USA, in press.

6. Cigan, A. M., M. Foiani, E. M. Hannig, and A. G. Hinnebusch. 1991. Complex formation by positive and negative translational regulators of GCN4. Mol. Cell. Biol. 11:3217-3228.

7. Cigan, A. M., E. K. Pabich, L. Feng, and T. F. Donahue. 1989. Yeast translation initiation suppressor sui2 encodes the $\alpha$ subunit of eukaryotic initiation factor 2 and shares identity with the human $\alpha$ subunit. Proc. Natl. Acad. Sci. USA 86:2784-2788.

8. De Benedetti, A., and C. Baglioni. 1983. Phosphorylation of initiation factor eIF $2 \alpha$, binding of mRNA to 48 S complexes, and its reutilization in initiation of protein synthesis. J. Biol. Chem. 258:14556-14562.

9. Dever, T. E., J.-J. Chen, G. N. Barber, A. M. Cigan, L. Feng, T. F. Donahue, I. M. London, M. G. Katze, and A. G. Hinnebusch. 1993. Mammalian eIF-2 $\alpha$ kinases functionally substitute for GCN2 in the GCN4 translational control mechanism of yeast. Proc. Natl. Acad. Sci. USA 90:4616-4620.

10. Dever, T. E., L. Feng, R. C. Wek, A. M. Cigan, T. D. Donahue, and A. G. Hinnebusch. 1992. Phosphorylation of initiation factor $2 \alpha$ by protein kinase $\mathrm{GCN} 2$ mediates gene-specific translational control of GCN4 in yeast. Cell 68:585-596.

11. Dieckmann, C. L., and A. Tzagolofi. 1985. Assembly of the mitochondrial membrane system. J. Biol. Chem. 260:1513-1520.

12. Foiani, M., A. M. Cigan, C. J. Paddon, S. Harashima, and A. G. Hinnebusch. 1991. GCD2, a translational repressor of the GCN4 gene, has a general function in the initiation of protein synthesis in Saccharomyces cerevisiae. Mol. Cell. Biol. 11:3203-3216.

13. Gross, M., R. Redman, and D. A. Kaplansky. 1985. Evidence that the primary effect of phosphorylation of eukaryotic initiation factor $2 \alpha$ in rabbit reticulocyte lysate is inhibition of the release of eukaryotic initiation factor 2-GDP from $60 \mathrm{~S}$ ribosomal subunits. J. Biol. Chem. 260:9491-9500.

14. Gross, M., M. Wing, C. Rundquist, and M. S. Rubino. 1987. Evidence that phosphorylation of eIF- $2 \alpha$ prevents the eIF-2Bmediated dissociation of eIF-2 GDP from the 60S subunit of complete initiation complexes. J. Biol. Chem. 262:6899-6907. 
15. Haffter, P., T. W. McMullin, and T. D. Fox. 1990. A genetic link between an mRNA-specific translational activator and the translational system in yeast mitochondria. Genetics 125:495-503.

16. Haffter, P., T. W. McMullin, and T. D. Fox. 1991. Functional interactions among two yeast mitochondrial ribosomal proteins and an mRNA-specific translational activator. Genetics 127: 319-326.

17. Hannig, E. M., N. P. Williams, R. C. Wek, and A. G. Hinnebusch. 1990. The translational activator GCN3 functions downstream from GCN1 and GCN2 in the regulatory pathway that couples GCN4 expression to amino acid availability in Saccharomyces cerevisiae. Genetics 126:549-562.

18. Hannig, E. M., A. M. Cigan, B. A. Freeman, and T. G. Kinzy. 1992. GCD11, a negative regulator of $G C N 4$ expression, encodes the gamma subunit of eIF-2 in Saccharomyces cerevisiae. Mol. Cell. Biol. 13:506-520.

19. Hannig, E. M., and A. G. Hinnebusch. 1988. Molecular analysis of $G C N 3$, a translational activator of GCN4: evidence for posttranslational control of $G C N 3$ regulatory function. Mol. Cell. Biol. 8:4808-4820.

20. Harashima, S., E. M. Hannig, and A. G. Hinnebusch. 1987. Interactions between positive and negative regulators of $G C N 4$ controlling gene expression and entry into the yeast cell cycle. Genetics 117:409-419.

21. Harashima, S., and A. G. Hinnebusch. 1986. Multiple $G C D$ genes required for repression of $G C N 4$, a transcriptional activator of amino acid biosynthetic genes in Saccharomyces cerevisiae. Mol. Cell. Biol. 6:3990-3998.

22. Hennessy, K. M., A. Lee, E. Chen, and D. Botstein. 1991. A group of interacting yeast DNA replication genes. Genes Dev. 5:958-969.

23. Hershey, J. W. B. 1991. Translational control in mammalian cells. Annu. Rev. Biochem. 60:717-755.

24. Hinnebusch, A. G. 1985. A hierarchy of trans-acting factors modulate translation of an activator of amino acid biosynthetic genes in yeast. Mol. Cell. Biol. 5:2349-2360.

25. Hinnebusch, A. G. 1988. Mechanisms of gene regulation in the general control of amino acid biosynthesis in Saccharomyces cerevisiae. Microbiol. Rev. 52:248-273.

26. Hinnebusch, A. G., and G. R. Fink. 1983. Positive regulation in the general amino acid control of Saccharomyces cerevisiae. Proc. Natl. Acad. Sci. USA 80:5374-5378.

27. Hinnebusch, A. G., and G. R. Fink. 1983. Repeated DNA sequences upstream from $H I S 1$ also occur at several other co-regulated genes in Saccharomyces cerevisiae. J. Biol. Chem. 258:5238-5247.

28. Hinnebusch, A. G., G. Lucchini, and G. R. Fink. 1985. A synthetic HIS4 regulatory element confers general amino acid control on the cytochrome $c$ gene (CYC1) of yeast. Proc. Natl. Acad. Sci. USA 82:498-502.

29. Hinnebusch, A. G., R. C. Wek, T. E. Dever, A. M. Cigan, L. Feng, and T. F. Donahue. 1993. Regulation of GCN4 expression in yeast: gene specific translational control by phosphorylation of eIF-2 $\alpha$, p. 87-115. In J. Ilan (ed.), Translational regulation of gene expression. Plenum Press, New York.

30. Ito, H., Y. Fukada, K. Murata, and A. Kimura. 1983. Transformation of intact yeast cells treated with alkali cations. J. Bacteriol. 153:163-168.

31. Konieczny, A., and B. Safer. 1983. Purification of the eukaryotic initiation factor 2-eukaryotic initiation factor $2 \mathrm{~B}$ complex and characterization of its guanine nucleotide exchange activity during protein synthesis initiation. J. Biol. Chem. 258:34023408 .

32. Koromilas, A. E., S. Roy, G. N. Barber, M. G. Katze, and N. Sonenberg. 1992. Malignant transformation by a mutant of the IFN-inducible dsRNA dependent protein kinase. Science 257: $1685-1688$.

33. Lanker, S., J. L. Bushman, A. G. Hinnebusch, H. Trachsel, and P. P. Mueller. 1992. Autoregulation of the Saccharomyces cerevisiae gene $G C D 5 / K R S 1$ encoding lysyl-tRNA synthetase by translational regulation of $G C N 4$ and transcriptional activation of GCD5. Cell 70:647-657.
34. London, I. M., D. H. Levin, R. L. Matts, N. S. B. Thomas, R. Petryshyn, and J. J. Chen. 1987. Regulation of protein synthesis, p. 359-380. In The enzymes. P. D. Boyer and E. G. Krebs (ed.), Academic Press, New York.

35. Lucchini, G., A. G. Hinnebusch, C. Chen, and G. R. Fink. 1984. Positive regulatory interactions of the HIS4 gene of Saccharomyces cerevisiae. Mol. Cell. Biol. 4:1326-1333.

36. Matts, R. L., D. H. Levin, and I. M. London. 1983. Effect of phosphorylation of the $\alpha$-subunit of eukaryotic initiation factor 2 on the function of reversing factor in the initiation of protein synthesis. Proc. Natl. Acad. Sci. USA 80:2559-2563.

37. McMullin, T. W., P. Haffter, and T. D. Fox. 1990. A novel small subunit ribosomal protein of yeast mitochondria that interacts functionally with an mRNA-specific translational activator. Mol. Cell. Biol. 10:4590-4595.

38. Moehle, C. M., and A. G. Hinnebusch. 1991. Association of RAP1 binding sites with stringent control of ribosomal protein gene transcription in Saccharomyces cerevisiae. Mol. Cell. Biol. 11:2723-2735.

39. Moldave, K. 1985. Eukaryotic protein synthesis. Annu. Rev. Biochem. 54:1109-1149.

40. Niederberger, P., M. Aebi, and R. Huetter. 1986. Identification and characterization of four new GCD genes in Saccharomyces cerevisiae. Curr. Genet. 10:657-664.

41. Novick, P., B. C. Osmond, and D. Botstein. 1989. Suppressors of yeast actin mutations. Genetics 121:659-674.

42. Paddon, C. J., E. M. Hannig, and A. G. Hinnebusch. 1989. Amino acid sequence similarity between GCN3 and GCD2, positive and negative translational regulators of GCN4: evidence for antagonism by competition. Genetics 122:551-559.

43. Paddon, C. J., and A. G. Hinnebusch. 1989. gcd12 mutations are gcn3-dependent alleles of $G C D 2$, a negative regulator of $G C N 4$ in the general amino acid control of Saccharomyces cerevisiae. Genetics 122:543-550.

44. Pain, V. M. 1986. Initiation of protein synthesis in mammalian cells. Biochem. J. 235:625-637.

45. Parent, S. A., C. M. Fenimore, and K. A. Bostian. 1985. Vector systems for the expression, analysis and cloning of DNA sequences in $S$. cerevisiae. Yeast 1:83-138.

46. Peterson, C. L., and I. Herskowitz. 1992. Characterization of the yeast $S W I 1, S W I 2$, and $S W I 3$ genes, which encode a global activator of transcription. Cell 68:573-583.

47. Rowlands, A. G., R. Panniers, and E. C. Henshaw. 1988. The catalytic mechanism of guanine nucleotide exchange factor action and competitive inhibition by phosphorylated eukaryotic initiation factor 2. J. Biol. Chem. 263:5526-5533.

48. Sherman, F., G. R. Fink, and C. W. Lawrence. 1979. Methods in yeast genetics. Cold Spring Harbor Laboratory, Cold Spring Harbor, N.Y.

49. Siekierka, J., V. Manne, and S. Ochoa. 1984. Mechanism of translational control by partial phosphorylation of the alpha subunit of eukaryotic initiation factor 2. Proc. Natl. Acad. Sci. USA 81:352-356.

50. Sikorski, R. S., and P. Hieter. 1989. A system of shuttle vectors and yeast host strains designed for efficient manipulation of DNA in Saccharomyces cerevisiae. Genetics 122:19-27.

51. Tamkun, J. W., R. Deuring, M. P. Scott, M. Kissinger, A. M. Pattatucci, T. C. Kaufman, and J. A. Kennison. 1992. brahma: a regulator of drosophila homeotic genes structurally related to the yeast transcriptional activator SNF2/SWI2. Cell 68:561-572.

52. Thomas, N. S. B., R. L. Matts, D. H. Levin, and I. M. London. 1985. The $60 \mathrm{~S}$ ribosomal subunit as a carrier of eukaryotic initiation factor 2 and the site of reversing factor activity during protein synthesis. J. Biol. Chem. 260:9860-9866.

53. Williams, N. P., A. G. Hinnebusch, and T. F. Donahue. 1989. Mutations in the structural genes for eukaryotic initiation factors $2 \alpha$ and $2 \beta$ of Saccharomyces cerevisiae disrupt translational control of GCN4 mRNA. Proc. Natl. Acad. Sci. USA 86:7515-7519.

54. Winston, F., F. Chumley, and G. R. Fink. 1983. Eviction and transplacement of mutant genes in yeast. Methods Enzymol. 101:211-227. 